\title{
Cardiac CaMKII $\delta$ and Wenxin Keli Prevents Ang II-Induced Cardiomyocyte Hypertrophy by Modulating CnA-NFATc4 and Inflammatory Signaling Pathways in H9c2 Cells
}

\author{
Na An, ${ }^{1,2}$ Yu Chen, ${ }^{3}$ Yanfen Xing, ${ }^{4}$ Honghua $W u,{ }^{5}$ Xiongyi Gao, ${ }^{1}$ Hengwen Chen, ${ }^{1}$ Ke Song, ${ }^{2}$ \\ Yuanyuan Li, ${ }^{2}$ Xinye Li, ${ }^{1,6}$ Fan Yang, ${ }^{1}$ Xiandu Pan, ${ }^{1,6}$ Xiaofang He, ${ }^{2}$ Xin Wang, ${ }^{2}$ Yang Li, \\ Yonghong Gao $\mathbb{D}^{2}{ }^{2}$ and Yanwei Xing $\mathbb{D}^{1}$ \\ ${ }^{1}$ Guang'anmen Hospital, Chinese Academy of Chinese Medical Sciences, Beijing 100053, China \\ ${ }^{2}$ Key Laboratory of Chinese Internal Medicine of Ministry of Education, \\ Dongzhimen Hospital Affiliated to Beijing University of Chinese Medicine, Beijing 100700, China \\ ${ }^{3}$ Fujian Health College, Fuzhou 350101, China \\ ${ }^{4}$ Shanxi University of Chinese Medicine, Jinzhong 030619, China \\ ${ }^{5}$ Tianjin State Key Laboratory of Modern Chinese Medicine, \\ Tianjin Key Laboratory of TCM Chemistry and Analysis Institute of Traditional Chinese Medicine, \\ Tianjin University of Traditional Chinese Medicine, Tianjin 300193, China \\ ${ }^{6}$ Beijing University of Chinese Medicine, Beijing, China \\ ${ }^{7}$ Department of Cardiology, General Hospital of People's Liberation Army, Beijing 100853, China
}

Correspondence should be addressed to Yonghong Gao; gaoyh7088@163.com and Yanwei Xing; xingyanwei12345@163.com

$\mathrm{Na}$ An, Yu Chen, Yanfen Xing, and Honghua Wu contributed equally to this work.

Received 26 July 2020; Revised 18 August 2020; Accepted 20 September 2020; Published 23 October 2020

Academic Editor: Hong Chang

Copyright (C) $2020 \mathrm{Na}$ An et al. This is an open access article distributed under the Creative Commons Attribution License, which permits unrestricted use, distribution, and reproduction in any medium, provided the original work is properly cited.

\begin{abstract}
Previous studies have demonstrated that calcium-/calmodulin-dependent protein kinase II (CaMKII) and calcineurin A-nuclear factor of activated T-cell (CnA-NFAT) signaling pathways play key roles in cardiac hypertrophy $(\mathrm{CH})$. However, the interaction between CaMKII and CnA-NFAT signaling remains unclear. H9c2 cells were cultured and treated with angiotensin II (Ang II) with or without silenced CaMKII $\delta$ (siCaMKII) and cyclosporine A (CsA, a calcineurin inhibitor) and subsequently treated with Wenxin Keli (WXKL). Patch clamp recording was conducted to assess L-type Ca ${ }^{2+}$ current $\left(\mathrm{I}_{\mathrm{Ca}-\mathrm{L}}\right)$, and the expression of proteins involved in signaling pathways was measured by western blotting. Myocardial cytoskeletal protein and nuclear translocation of target proteins were assessed by immunofluorescence. The results indicated that siCaMKII suppressed Ang II-induced CH, as evidenced by reduced cell surface area and $\mathrm{I}_{\mathrm{Ca}-\mathrm{L}}$. Notably, siCaMKII inhibited Ang II-induced activation of CnA and NFATc4 nuclear transfer. Inflammatory signaling was inhibited by siCaMKII and WXKL. Interestingly, CsA inhibited CnA-NFAT pathway expression but activated CaMKII signaling. In conclusion, siCaMKII may improve CH, possibly by blocking CnA-NFAT and MyD88 signaling, and WXKL has a similar effect. These data suggest that inhibiting CaMKII, but not CnA, may be a promising approach to attenuate $\mathrm{CH}$ and arrhythmia progression.
\end{abstract}

\section{Introduction}

Cardiomyocyte hypertrophy $(\mathrm{CH})$ is an adaptive response to the pathological stimuli that maintain normal cardiac function. $\mathrm{CH}$ is a prerequisite marker of heart failure (HF) and usually occurs after myocardial infarction and stress overload. Although the initially adaptive response can maintain cardiac output, sustained hypertrophic growth can lead to a pathological state that leads to decreased compliance, $\mathrm{HF}$, and sudden death [1-5]. Therefore, $\mathrm{CH}$ remains 
a major threat in the population, and it is necessary to elucidate the pivotal molecular mechanisms involved in $\mathrm{CH}$ that can ameliorate pathological $\mathrm{CH}$ responses. Different signaling molecules have been considered as causes of myocardial hypertrophy, including nuclear factor of activated $\mathrm{T}$ cells (NFAT), calcium-/calmodulin- (CaM-) dependent protein kinase II (CaMKII), and $\beta$-adrenergic receptors [6].

Calcineurin (CaN), a calcium- and CaM-dependent serine/threonine phosphatase, is a well-established mediator of $\beta$-adrenergic-induced $\mathrm{CH}[7,8]$. CaN consists of two subunits: the catalytic subunit $(\mathrm{CnA})$ and a regulatory $\mathrm{Ca}^{2+}$-binding subunit $(\mathrm{CnB})[9,10]$. It is generally known that $\mathrm{CaN}$ plays an influential role in regulating pathological hypertrophy. The constitutive activation of $\mathrm{CaN}$ and its downstream target, NFAT, are thought to play an important role in abnormal $\mathrm{CH}[11,12]$. Moreover, it is known that CaMKII phosphorylates many vital signaling factors that are related to initiating abnormal hypertrophy [13]. Transgenic overexpression of the splice variants CaMKII $\delta \mathrm{b}$ (located in the nucleus) and CaMKII $\delta \mathrm{c}$ (located in the cytosol) promotes $\mathrm{CH}$ and dilated cardiomyopathy, respectively. However, the complete pathological molecular mechanism is not fully understood, which hinders the development of improved treatments for $\mathrm{CH}[14,15] . \mathrm{Ca}^{2+}$ dependent signaling through CaMKII and $\mathrm{CaN}$ has been suggested to contribute to adverse $\mathrm{CH}$ [16]. However, the interaction between CaMKII and CnA-NFAT signaling for $\mathrm{CH}$ remains unclear.

Wenxin Keli (WXKL) is the first Chinese medicine approved by the Chinese state for its antiarrhythmic effects; the medicine can tonify $q_{i}$, supply $y_{\text {in }}$, promote blood circulation, and remove blood stasis according to the theory of traditional Chinese medicine. The main components of WXKL consist of Nardostachys jatamansi (D.Don) DC (Gansong), Codonopsis pilosula (Franch.) Nannf (Dangshen), Panax notoginseng (Burkill) F.H.Chen (Sanqi), Succinum (Hupo), and Polygonatum cyrtonema Hua (Huangjing). According to the national pharmacopoeia [17], WXKL mainly contains notoginseng saponin $\mathrm{R} 1\left(\mathrm{C}_{47} \mathrm{H}_{80} \mathrm{O}_{18}\right)$, ginseng saponin $\mathrm{Rg} 1$ $\left(\mathrm{C}_{42} \mathrm{H}_{72} \mathrm{O}_{14}\right)$, and ginseng saponin $\mathrm{Rb} 1\left(\mathrm{C}_{54} \mathrm{H}_{92} \mathrm{O}_{23}\right)$. Figure 1 [18] displays the HPLC chromatograms of the chemical reference substances, WXKL, and negative samples of Panax notoginseng and Codonopsis pilosula. Previous studies have shown that WXKL may inhibit HF and arrhythmia by regulating the CaMKII signaling pathway [19-23]. WXKL in the treatment of patients with HF or arrhythmia can improve the exercise tolerance and is beneficial for the recovery of cardiac function [24-26]. However, to the best of our knowledge, whether WXKL improves $\mathrm{CH}$ via regulating the CaMKII and CnA-NFAT signaling pathways has not been investigated.

Given the pivotal roles of the CaMKII and CaN signaling pathways in the regulation of abnormal hypertrophy, we selected H9c2 cells induced by angiotensin II (Ang II) and used CaMKII silencing and treatment with cyclosporine A (CsA, a CaN inhibitor) to explore the correlation between the CaMKII and CnA-NFAT signaling pathways. Additionally, we sought to determine whether WXKL could improve HF by regulating CaMKII and CnA-NFAT.

\section{Materials and Methods}

2.1. Construction of Silenced CaMKIIS in H9c2-1632 Cells. H9c2 rat embryonic cardiomyocyte cells were obtained from the Institute of Basic Medical Sciences, Chinese Academy of Medical Sciences (Cell Resource Center, IBMS, CAMS/ PUMC, China). First, RNA interference at the site of the target gene, CaMKII $\delta$ (Rat), was performed. According to the target gene sequence site, four sequences with the target gene were inserted into the vector pCDNA6.2 emGFP, and miRNA reverse primer was used as the sequencing primer (Table 1). Sequencing results verified that the correct sequence was inserted. Transfection and preparation of plasmids, blasticidin screening, and determination of the concentration of $\mathrm{H} 9 \mathrm{c} 2$ cells, and finally cell transfection and screening were performed. Real-time PCR results showed that the relative mRNA expression of H9c2-1141 and H9c21632 sites was significantly reduced after RNA interference (Figure S2A), and the inhibition rate of relative mRNA expression after $\mathrm{H} 9 \mathrm{c} 2-1632$ site interference was slightly lower than that of $\mathrm{H} 9 \mathrm{c} 2-1141$, but the difference was not statistically significant (Table 2 ). CaMKII $\delta$ protein expression was determined by western blot analysis, and the results showed that, after performing RNA interference at four sites, CaMKII $\delta$ protein expression was decreased, with the greatest decrease at the $\mathrm{H} 9 \mathrm{c} 2-1632$ site (Figure S2B). Combined with real-time PCR, the H9c2-1632 site was finally selected as a target to construct a CaMKII $\delta$ (Rat) RNA interference cell line. The H9c2-1632 cells were cultured in an incubator containing $5 \% \mathrm{CO}_{2}$ in high-sugar Dulbecco's modified Eagle's medium containing $10 \%$ fetal bovine serum at $37^{\circ} \mathrm{C}$.

2.2. Drugs and Solutions. Ang II (A9525, Sigma Co., St. Louis, MO, USA) was dissolved in deionized water at a concentration of $10^{-5} \mathrm{~mol} / \mathrm{L} ; 20 \mu \mathrm{L}$ of this solution was added to the culture medium $(2 \mathrm{~mL})$, reaching a final concentration of $10^{-7} \mathrm{~mol} / \mathrm{L}$. CsA (Sigma Co.) was dissolved in ethyl alcohol at a concentration of $10^{-4} \mathrm{~mol} / \mathrm{L} ; 20 \mu \mathrm{L}$ of this solution was added to the culture medium $(2 \mathrm{~mL})$ at a final concentration of $10^{-6} \mathrm{~mol} / \mathrm{L}$. WXKL (1910051, Shandong Buchang Pharmaceuticals Co., Ltd., Shandong, China) was dissolved in saline at a concentration of $5 \mathrm{~g} / \mathrm{L}$.

In HPLC, $1 \mathrm{~g}$ of finely ground WXKL was added to a conical flask with a stopper, $50 \mathrm{ml}$ of water-saturated $n$ butanol was added, and the flask was tightly stoppered and weighed. After soaking for 12 hours and ultrasonic treatment (power $300 \mathrm{~W}$, frequency $40 \mathrm{kHz}$ ) for $1 \mathrm{~h}$, the flask was weighed again after cooling, water-saturated $n$-butanol was added to make up for the lost weight, and the flask was shaken well. After filtration, $25 \mathrm{ml}$ of the filtrate was collected. The $n$-butanol solution was evaporated to dryness, and methanol solution was added to the residue to $10 \mathrm{ml}$ and shaken. According to the proportion of prescription Chinese medicine, notoginseng and Codonopsis were removed separately to make negative samples of Panax notoginseng and Codouopsis pilosula, and the negative control solution was prepared according to the previously mentioned methods. 


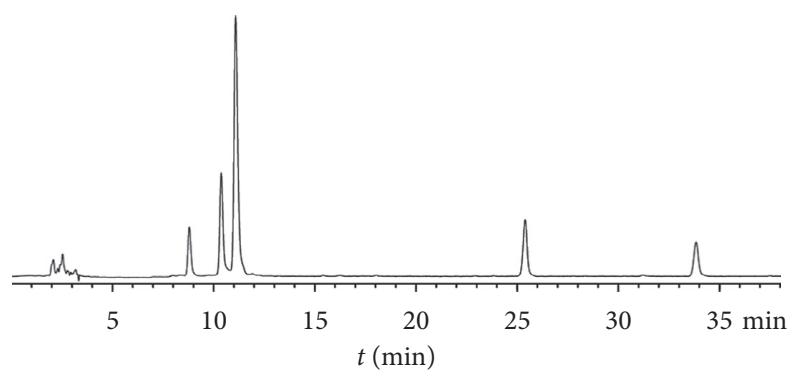

(a)

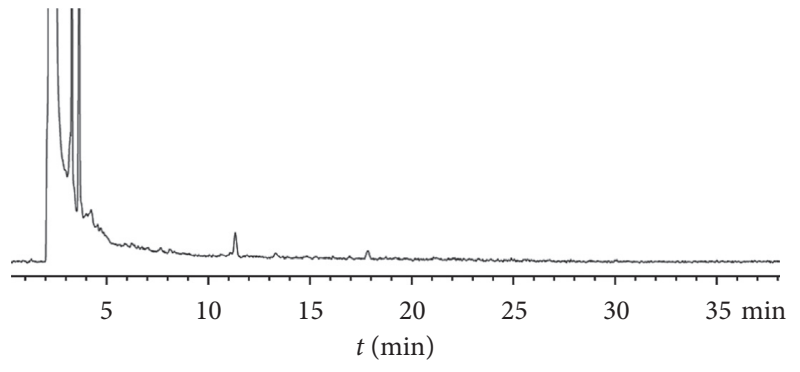

(c)

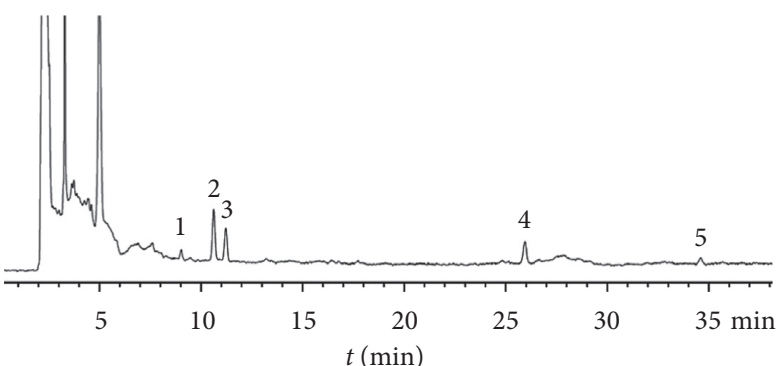

(b)

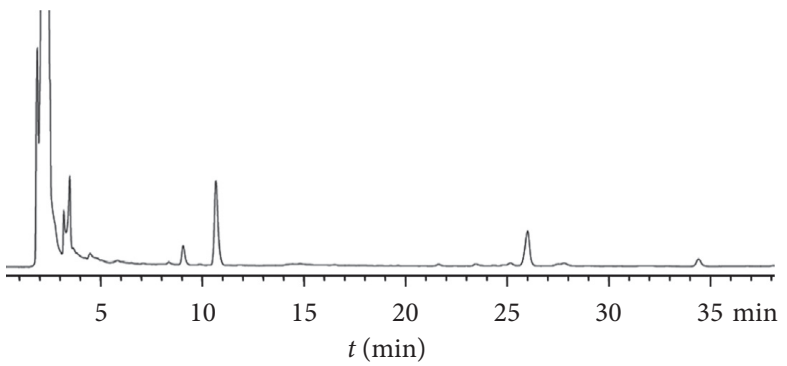

(d)

FIgURE 1: The HPLC chromatograms of WXKL. (a) Chemical reference substances, (b) WXKL, (c) negative samples of Panax notoginseng, and (d) Codouopsis pilosula. (1) notoginsenoside R1; (2) ginsenoside Rg1; (3) obetyolin; (4) ginsenoside Rb1; (5) ginsenoside Rd.

TABLE 1: CaMKII $\delta$ (Rat) (NM_012519.2) RNAi site.

\begin{tabular}{lcc}
\hline & Initiation site & RNAi site sequence \\
\hline $1 \#$ & 408 & TAGAATCTGCCGTCTCTTGAA \\
$2 \#$ & 754 & CCTGGGTATCTTTCTCCAGAA \\
$3 \#$ & 1141 & ACTATGCTGGCTACGAGAAAT \\
$4 \#$ & 1632 & GTACATGGATGGAAATGGAAT \\
\hline
\end{tabular}

TABLE 2: Inhibition rate of CaMKII $\delta$ (rat) H9c2-1141 and H9c21632 sites after the RNA interference.

\begin{tabular}{lcc}
\hline Cells & $\begin{array}{c}\text { Relative } \\
\text { expression }\end{array}$ & Inhibition rate of CaMKII $\delta$ (rat) \\
\hline H9c2- & 1 & 0 \\
NEG & & $82.34 \%$ \\
H9c2-1141 & 0.1765726 & $81.10 \%$ \\
H9c2-1632 & 0.1890273 & \\
\hline
\end{tabular}

Methanol was added to prepare reference substances containing notoginsenoside R1 $0.247 \mathrm{mg}$, ginsenoside $\mathrm{Rg} 1$ $0.422 \mathrm{mg}$, ginsenoside $0.042 \mathrm{mg}$, ginsenoside $\mathrm{Rb} 10.847 \mathrm{mg}$, and ginsenoside Rd $0.255 \mathrm{mg}$ per $1 \mathrm{~mL}$.

2.3. Chromatographic Conditions. Analyses were performed on an Agilent 1260 HPLC system consisting of a quaternary delivery system, an autosampler, and a DAD detector. All the separations were carried out on Amethyst $\mathrm{C}_{18}$ column $(4.6 \times 250 \mathrm{~mm}, 4 \mu \mathrm{m})$. The gradient elution used acetonitrile (A) and water (B) as a mobile phase at a flow rate of $1 \mathrm{~mL} /$ min. The gradient program was as follows: $0-14 \mathrm{~min}, 22 \%-$ $30 \% \mathrm{~A} ; \quad 14-35 \mathrm{~min}, 30 \%-38 \% \mathrm{~A} ; 35-45 \mathrm{~min}, 38 \%-38 \% \mathrm{~A}$; 45-47 min, 38\%-95\%A; 47-62 min, 95\%-95\%A; 62-65 min, $95 \%-22 \% \mathrm{~A}$. The column temperature was maintained at $27^{\circ} \mathrm{C}$, and the chromatogram was monitored at a wavelength of $210 \mathrm{~nm}$.

2.4. Cell Grouping and Drug Administration. H9c2 rat embryonic cardiomyocytes were split into 13 different treatment groups: (1) negative control group: only secondary antibody was added to H9c2 cells with no primary antibody; (2) control group: H9c2 cells were cultured for $72 \mathrm{~h}$; (3) control + Ang II group: H9c2 cells were pretreated with Ang II for $48 \mathrm{~h}$ and cultured for another $24 \mathrm{~h}$; (4) control + Ang II + WXKL group: $\mathrm{H} 9 \mathrm{c} 2$ cells were pretreated with Ang II for $48 \mathrm{~h}$, treated with WXKL, and cultured for another $24 \mathrm{~h}$; (5) control + WXKL group: H9c2 cells were cultured for $48 \mathrm{~h}$, treated with WXKL, and cultured for another $24 \mathrm{~h}$; (6) siCaMKII group: H9c21632 cells were cultured for $72 \mathrm{~h}$; (7)siCaMKII + Ang II group: H9c2-1632 cells were pretreated with Ang II for $48 \mathrm{~h}$ and cultured for another $24 \mathrm{~h}$; (8) siCaMKII + Ang II + WXKL group: H9c2-1632 cells were pretreated with Ang II for $48 \mathrm{~h}$, treated with WXKL, and cultured for another $24 \mathrm{~h}$; (9) siCaMKII + WXKL group: H9c2-1632 cells were cultured for $48 \mathrm{~h}$, treated with WXKL, and cultured for another $24 \mathrm{~h}$; (10) CsA group: H9c2 cells were pretreated with CsA for $72 \mathrm{~h}$; (11) CsA + Ang II group: H9c2 cells were pretreated with CsA and Ang II for $48 \mathrm{~h}$ and cultured for another $24 \mathrm{~h}$; (12) CsA + Ang II + WXKL group: H9c2 cells were pretreated with CsA and Ang II for $48 \mathrm{~h}$, treated with WXKL, and cultured for another $24 \mathrm{~h}$; (13) CsA + WXKL group: H9c2 cells were pretreated with CsA for $48 \mathrm{~h}$, treated with WXKL, and cultured for another $24 \mathrm{~h}$.

2.5. Western Blot Analysis. Proteins were extracted from H9c2 cells, subsequently lysed with lysis buffer containing phenylmethylsulfonyl fluoride in ice for $40 \mathrm{~min}$, 
and shaken once every $8 \mathrm{~min}$ during this period. The lysates were centrifuged at $1200 \mathrm{rpm}$ for $20 \mathrm{~min}$ at $4^{\circ} \mathrm{C}$, and the supernatants were collected. Proteins were analyzed by sodium dodecyl sulfate-polyacrylamide gel electrophoresis, transferred onto nitrocellulose membranes, blocked in $5 \%$ bovine serum albumin (BSA) or milk, and then incubated with primary antibodies at $4^{\circ} \mathrm{C}$ overnight. Subsequently, the membranes were washed three times with tris-buffered saline/Tween 20 at the specified time intervals and finally incubated with secondary antibody at room temperature. ECL visualization was performed, and the resulting images were captured using the GeneGnome Gel Imaging System (Syngene Co., Bangalore, India). The gel images were analyzed using ImageJ software (Image-Pro Plus, Media Cybernetics, Rockville, MD, USA). The antibodies used in the present study are listed in Table S1.

2.6. Confocal Imaging. For the cytoskeletal assay, H9c2 cells were cultured in a confocal laser culture dish until reaching a moderate density. Upon reaching $70-80 \%$ confluence, the cells were washed with phosphate-buffered saline (PBS) twice, fixed with $4 \%$ paraformaldehyde for $15 \mathrm{~min}$, and washed with PBS three times for $5 \mathrm{~min}$ each. Cells were incubated with $0.1 \%$ Triton X-100 penetrating fluid at room temperature for $5 \mathrm{~min}$ and washed thrice with PBS for 5 min each. Rhodamine-phalloidin was diluted with PBS $(2.5 \mu \mathrm{L})$ and added to $200 \mu \mathrm{L}$ of the working fluid, fixed for $20 \mathrm{~min}$, and washed with PBS three times for $5 \mathrm{~min}$ each. Cells were subjected to confocal laser microscopy. The cells of each group were removed and washed with cold PBS (precooled at $4^{\circ} \mathrm{C}$ ) twice, and the residual PBS was aspirated. Each group was fixed with $4 \%$ paraformaldehyde for $10 \mathrm{~min}$ at $-20^{\circ} \mathrm{C}$ and washed twice with PBS. Cells were permeabilized with $0.25 \%$ Triton X-100 for $10 \mathrm{~min}$ and washed three times with cold PBS for 5 min each wash and then treated with $1 \%$ BSA for 30 min to block nonspecific antigen recognition. Primary antibodies were diluted with $1 \%$ BSA in PBS and added at $100 \mu \mathrm{L}$ to each group. Cells were plated at $4^{\circ} \mathrm{C}$ for $12 \mathrm{~h}$ and then washed thrice with PBS for $5 \mathrm{~min}$ each. The secondary antibody was diluted with $1 \%$ BSA in PBS with $100 \mu \mathrm{L}$ per sample at room temperature for $60 \mathrm{~min}$ away from light. The secondary antibody was removed and cells were washed thrice with PBS for 5 min each. Cells were incubated with DAPI for nuclear staining; finally, an antifluorescence quencher was added. Later, a transparent nail polish seal was used, and cells were observed using a confocal microscope.

For measuring the nuclear translocation of NFATc4, the cell processing method is basically the same as previously mentioned. Images were analyzed using ImagePro Plus Analysis Software. First, color images were converted to gray images. The ratio of IOD to the area represented by NFATc4 fluorescence was measured. NFATc4 translocation was quantified as the ratio between the number of cells containing nucleus-localized NFATc4 (NFATc4-positive), and the total number of cells was counted.
2.7. Electrophysiological Recording. Using the whole-cell patch clamp technique, the whole-cell $\mathrm{Ca}^{2+}$ current was recorded by an Axon-700B amplifier (Axon Instruments, San Jose, CA, USA) with the pCLAMP 9.2 software (Axon Instruments). Borosilicate glass microelectrodes had tip resistances of 3.0-5.0 $\mathrm{M} \Omega$, which adjusts the three-dimensional manipulator for $\mathrm{G} \Omega$ sealing and breaks the membrane absorption in the whole-cell recording mode. The membrane capacitance and $\mathrm{I}_{\mathrm{Ca}-\mathrm{L}}$ current were recorded after stabilization. To eliminate the errors resulting from cell size, the I value was expressed as the current density $(\mathrm{pA} / \mathrm{pF})$. Cells were superfused with extracellular fluid containing (all in mmol/L): $125 \mathrm{NaCl}, 10.8 \mathrm{BaCl}_{2}, 1 \mathrm{MgCl}_{2}, 5.4 \mathrm{CsCl}, 10$ HEPES, and 10 glucose (pH 7.35, adjusted with $\mathrm{NaOH}$ ). A pipette solution was used containing $(\mathrm{mmol} / \mathrm{L}): 120 \mathrm{CsCl}, 3$ $\mathrm{MgCl}_{2}, 5 \mathrm{Na}_{2} \mathrm{ATP}, 10$ EGTA, and 5 HEPES ( $\mathrm{pH} 7.3$, adjusted with $\mathrm{CsOH}$ ).

2.8. Statistical Analysis. Data were expressed as mean \pm SD. One-way ANOVA was used to compare multiple groups with a normal distribution. Statistical analysis was performed using the SPSS program (version 20.0). $P<0.05$ was considered statistically significant, and $P<0.01$ was considered highly statistically significant. Data acquisition and analysis were performed using pCLAMP 9.2 software (Axon Instruments), Origin 6.1 software (MicroCal Software, Northampton, MA, USA), and GraphPad Prism 5 (GraphPad Software Incorporate, La Jolla, CA, USA).

\section{Results}

We explored the interaction between CaMKII and CnANFAT signaling in $\mathrm{CH}$ induced by Ang II and the effect of WXKL. Therefore, we sought to characterize the role of CaMKII and CnA-NFAT signaling in cardiomyocytes using siRNA-mediated silencing (siCaMKII) and CsA. We analyzed the results of cytoskeletal enlargement; L-type $\mathrm{Ca}^{2+}$ current $\left(\mathrm{I}_{\mathrm{Ca}-\mathrm{L}}\right)$; expression of CaMKII, CnA-NFAT, and inflammatory signaling pathways in cardiomyocytes induced by Ang II; and the protein expression and nuclear transfer of NFATc4.

\subsection{Effects of SiCaMKII and CsA on Myocardial Cytoskeletal} Protein. We used fluorescence confocal microscopy to observe cytoskeletal enlargement (a direct indicator of hypertrophy in myocardial cells) to assess whether the $\mathrm{CH}$ model had been successfully established and ensure the feasibility of the experiment.

Immunofluorescence staining showed that Ang II effectively induced $\mathrm{CH}$, as evidenced by an increase in cell width and length (Figure 2). CsA and siCaMKII prevented an Ang II-stimulated increase in cell size, and the effect was similar in cells treated with WXKL (Figure 2). Taken together, the results revealed that siCaMKII and CsA may inhibit the hypertrophic response to Ang II in H9c2 cells. Moreover, WXKL improved Ang II-induced $\mathrm{CH}$. 

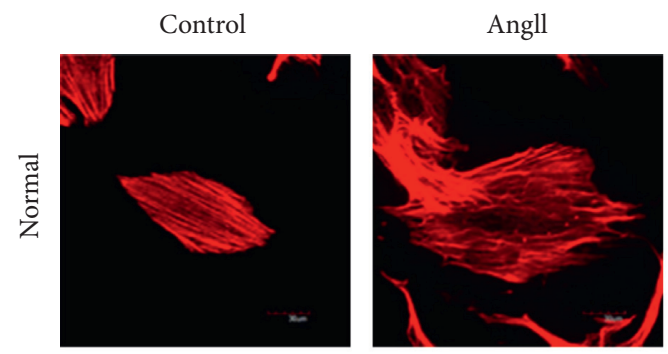

Angll + WXKL
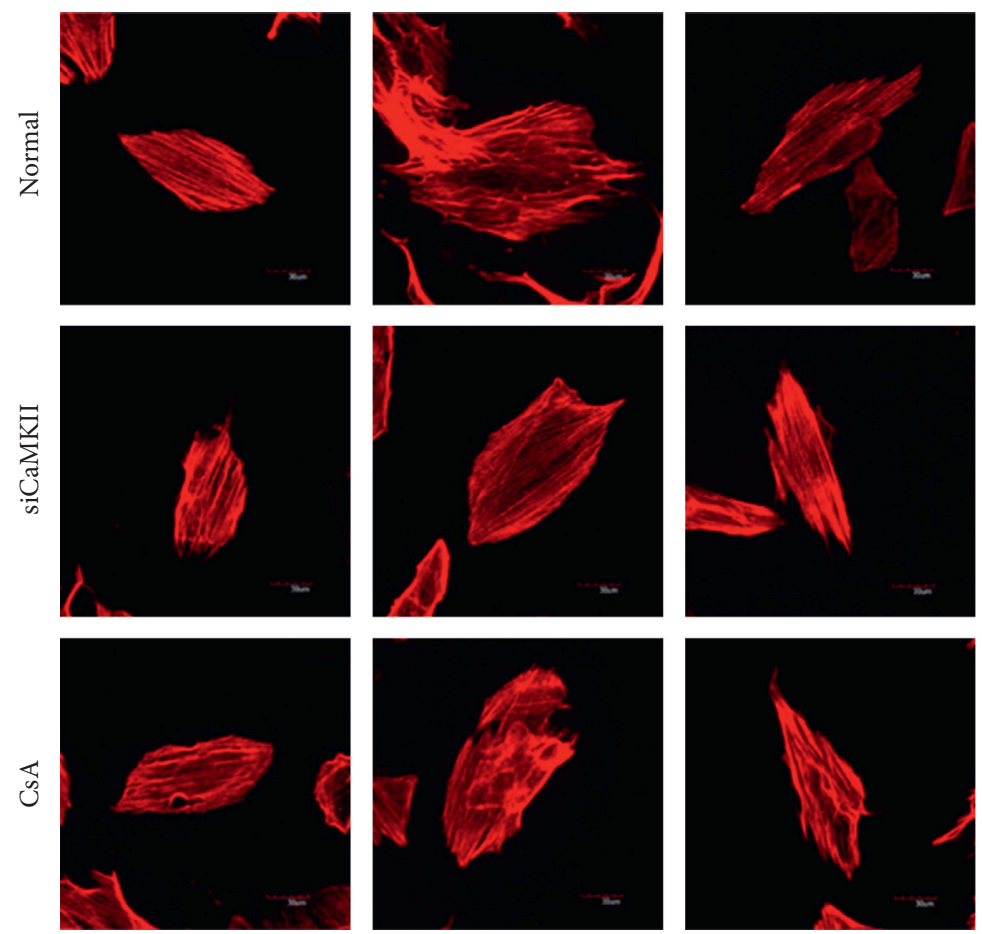
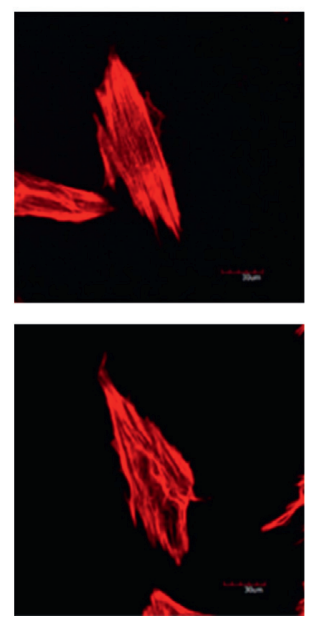

(a)

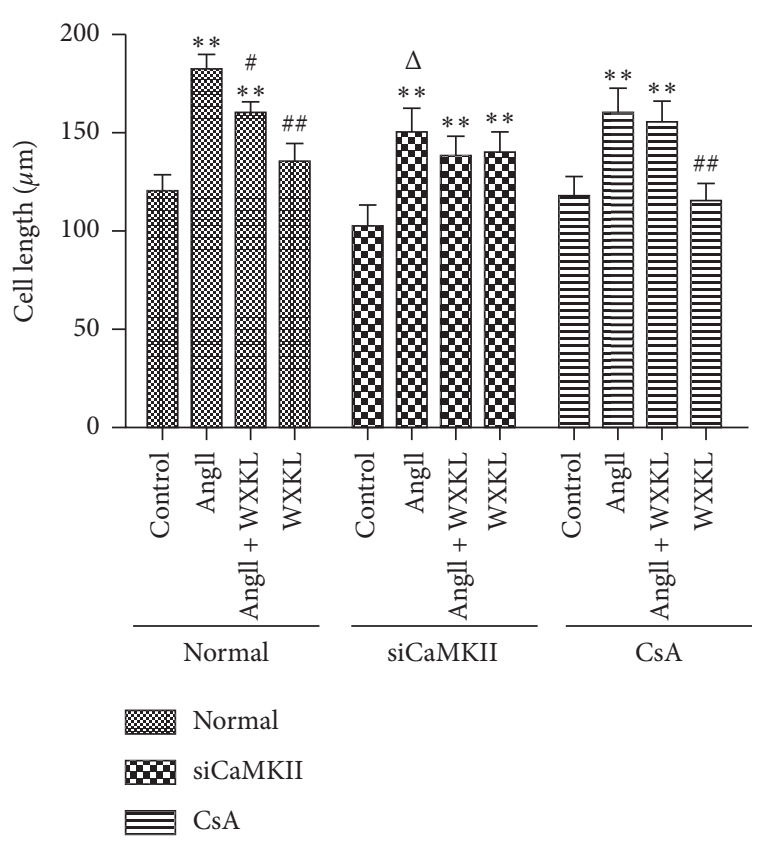

(b)

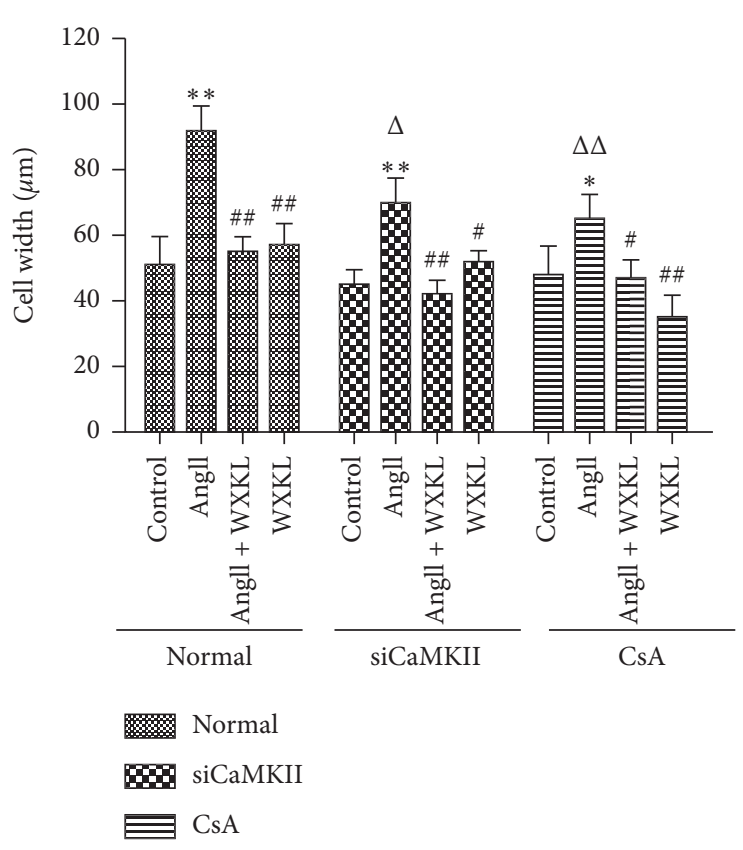

(c)

FIgUre 2: SiCaMKII decreases Ang II-induced cell surface area enlargement in H9c2 cells. H9c2 cells were treated with Ang II (10 ${ }^{-7} \mathrm{M}$ for $48 \mathrm{~h})$. CaMKII was silenced or CsA $\left(10^{-6} \mathrm{M}\right)$ was added to the culture medium prior to Ang II administration. After administering Ang II, WXKL ( $5 \mathrm{~g} / \mathrm{L}$ ) was added, and the culture medium was incubated for $24 \mathrm{~h}$. The control cells received no treatment. (a) Representative images of immunofluorescence staining for phalloidin following treatment ( $n=10$ cells per group). Scale bar: $30 \mu \mathrm{m}$. (b) Mean cell length measurement ( $n=10$ cells per group). (c) Mean cell width measurement ( $n=10$ cells per group). Data are presented as the mean \pm SD. Statistical significance was determined by one-way ANOVA. ${ }^{*} P<0.05$ and ${ }^{* *} P<0.01$ vs. the control group. ${ }^{\#} P<0.05$ and ${ }^{\# \#} P<0.01$ vs. the Ang II group. ${ }^{\wedge} P<0.05$ and ${ }^{\mathbf{\Delta}} P<0.01$; the control group in SiCaMKII or CsA vs. the control group in normal. ${ }^{\triangle} P<0.05$ and ${ }^{\triangle} P<0.01$; the Ang II group in SiCaMKII or CsA vs. the Ang II group in normal. 
3.2. $I_{C a-L}$ Significantly Reduced after CaMKIIS Silencing. $\mathrm{I}_{\mathrm{Ca}-\mathrm{L}}$ is present in many cardiomyocytes, and it plays a key role in the formation of an action potential plateau, intracellular $\mathrm{Ca}^{2+}$ elevation, and muscle contraction. $\mathrm{CaM}$, as a $\mathrm{Ca}^{2+}$ receptor, plays a major role in $\mathrm{Ca}^{2+}$-dependent inactivation and facilitation of $\mathrm{I}_{\mathrm{Ca}-\mathrm{L}}$.

Results revealed that compared with control treatment, Ang II significantly increased the amplitude of $\mathrm{I}_{\mathrm{Ca}-\mathrm{L}}$ within the range of $-20 \mathrm{mV}$ to $+10 \mathrm{mV}$. Pretreatment with siCaMKII reduced the elevated $\mathrm{I}_{\mathrm{Ca}-\mathrm{L}}$ amplitude. Similarly, WXKL decreased the Ang II-induced elevated amplitude of $\mathrm{I}_{\mathrm{Ca}-\mathrm{L}}$ (Figures 3(a) and 3(b)). Interestingly, in the CsA group (Figure 3), $\mathrm{I}_{\mathrm{Ca}-\mathrm{L}}$ was significantly increased, and its steadystate activation and inactivation were increased (Figures 3(c) and $3(\mathrm{~d})$ ). These results suggest that siCaMKII and WXKL inhibit the calcium current and impede inactivation. Furthermore, treatment with CsA led to the opposite results. Therefore, we suspected that CaN inhibition may alter other pathways and cause an increase in calcium current.

3.3. Treatment with CsA Activates the CaMKII Signaling Pathway. To further investigate Ang II-induced CaMKII expression in $\mathrm{H} 9 \mathrm{c} 2$ cells and the effect of drug intervention on CaMKII protein expression, we measured CaMKII protein levels using laser scanning confocal microscopy. Additionally, CaMKII signaling pathway proteins were subjected to western blotting to explore the mechanism.

The results revealed that Ang II treatment significantly increased the fluorescence intensity of CaMKII in H9c2 cells compared with control treatment (Figure 4(a)). Pretreatment with siCaMKII reduced the Ang II-induced elevation in the fluorescence intensity of CaMKII, and WXKL led to a similar decrease. However, the fluorescence intensity corresponding to the CaMKII protein level was significantly higher in the CsA group than in the normal group (Figure 4(b)) and was observed to decrease after WXKL treatment. Therefore, the results showed that the fluorescence intensity indicating CaMKII protein expression increased after stimulation with Ang II, whereas WXKL treatment significantly reduced this increase. However, treatment with CsA did not inhibit this effect.

The expression of CaMKII, p-CaMKII, RyR2, p-RyR2, PLB, and p-PLB in H9c2 cells after stimulation with Ang II for $48 \mathrm{~h}$ was evaluated using western blot analysis. Exposure of H9c2 cells to Ang II resulted in increased expression of CaMKII pathway proteins (Figure 5(a)). Pretreatment with siCaMKII significantly decreased the Ang II-induced elevated expression of p-RyR2 and p-PLB $(P<0.01$, Figures 5(e) and 5(f)) and reduced that of CaMKII and p-CaMKII $(P<0.05$, Figures 5(b) and 5(c)). Interestingly, pretreatment with CsA led to significantly upregulated CaMKII expression levels $(P<0.01$, Figure 5(b)). After treatment with WXKL for $24 \mathrm{~h}$, protein expression was decreased in each group. Therefore, the CaMKII signaling pathway was blocked by silencing CaMKII expression, which reduced the degree of hypertrophy, and WXKL had a similar effect. However, the CaMKII signaling pathway was activated in $\mathrm{H} 9 \mathrm{c} 2$ cells treated with CsA.
3.4. CaMKII Controls the CnA-NFAT Pathway. Next, we elucidated the effects of siCaMKII, CsA, and WXKL on the CnA-NFAT signaling pathway.

Treatment with Ang II significantly increased the fluorescence intensity of $\mathrm{CnA}$ in both the nucleus and the cytoplasm (Figure S1). The siCaMKII cells showed downregulation of $\mathrm{CnA}$ protein levels and lower fluorescence intensities. After treatment with WXKL for $24 \mathrm{~h}$, Ang II-induced CnA protein expression was inhibited, and its fluorescence intensity and nuclear transfer were reduced (Figure S1). These results revealed that the fluorescence intensity and nuclear transfer of $\mathrm{CnA}$ in the Ang II-treated group were significantly higher; however, CaMKII silencing as well as treatment with CsA and WXKL suppressed this effect.

As shown in Figure 6(a), the expression of upstream and downstream proteins, including $\mathrm{CnA}, \mathrm{p}-\mathrm{CnA}$, NFATc4, p-NFATc4, GATA4, p-GATA4, ANP, and BNP, in the CnANFAT signaling pathway was detected by western blotting. Exposure of H9c2 cells to Ang II resulted in increased expression of CnA-NFAT signaling pathway proteins (Figure 6). Pretreatment with siCaMKII markedly decreased the Ang II-induced elevated expression of p-CnA $(P<0.01$, Figure 6(c)) and reduced that of GATA4 and BNP $(P<0.05$, Figures 6(f) and 6(i)). Interestingly, pretreatment with CsA led to significantly upregulated ANP expression levels $(P<0.01$, Figure 6(h)). After treatment with WXKL for $24 \mathrm{~h}$, protein expression was decreased in each group. Therefore, the CnA-NFAT signaling pathway was blocked by silencing CaMKII expression, and WXKL played a role in improving the expression of various proteins in $\mathrm{CH}$.

3.5. SiCaMKII Inhibits Nuclear Transfer of NFATc4 in Hypertrophic Cardiomyocytes. Subsequently, we further analyzed CnA-NFAT signaling, and immunofluorescence and western blots were performed to detect NFATc4 nuclear translocation.

The results demonstrated that NFATc4 was translocated to the nucleus in response to Ang II stimulation (Figure 7), whereas pretreatment with siCaMKII or CsA inhibited Ang II-induced nuclear translocation of NFATc4; furthermore, such transfer was inhibited after treatment with WXKL. The data presented in Figures 6 and 7 suggest that inhibition of hypertrophy by siCaMKII and WXKL was mediated, at least in part, by CaMKII and CnA-NFATc4 signaling.

3.6. Effects of SiCaMKII and CsA on the Inflammatory Signaling Pathway (MyD88-TLR4). Finally, to further elucidate the interaction between the CaMKII and CnA-NFAT signaling pathways, western blotting was used to measure the expression of MyD88, NF- $\kappa \mathrm{B}, \mathrm{p}-\mathrm{NF}-\kappa \mathrm{B}, \mathrm{TLR} 2$, and TLR4 in different groups (Figure 8).

The results demonstrated that protein expression was increased in $\mathrm{H} 9 \mathrm{c} 2$ cells after Ang II treatment for $48 \mathrm{~h}$. A statistically significant difference was observed in the expression of MyD88, NF- $\kappa \mathrm{B}$, and p-NF- $\kappa \mathrm{B} \quad(P<0.01$, Figures $8(\mathrm{~b}), 8(\mathrm{c})$, and $8(\mathrm{~d}))$. Compared with that after Ang II treatment in normal $\mathrm{H} 9 \mathrm{c} 2$ group, inflammation-related 

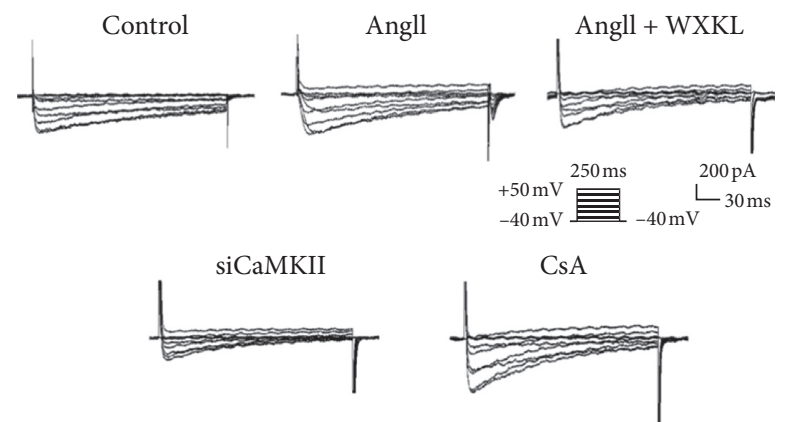

(a)
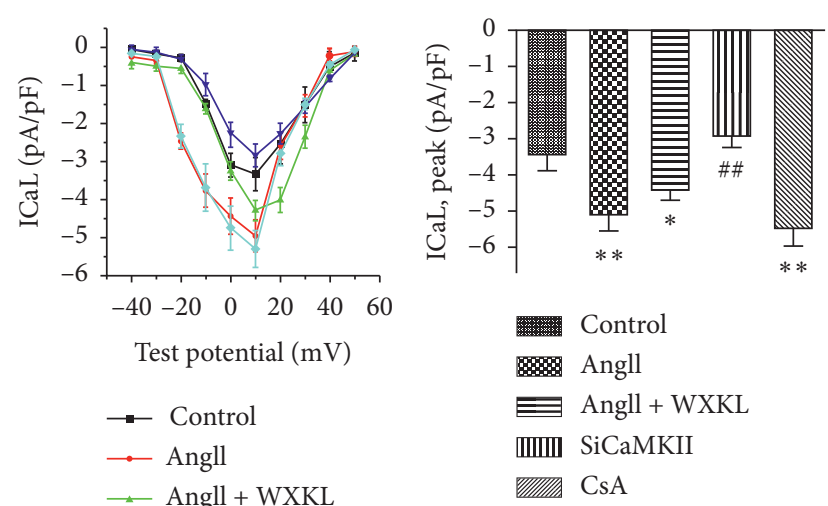

(b)
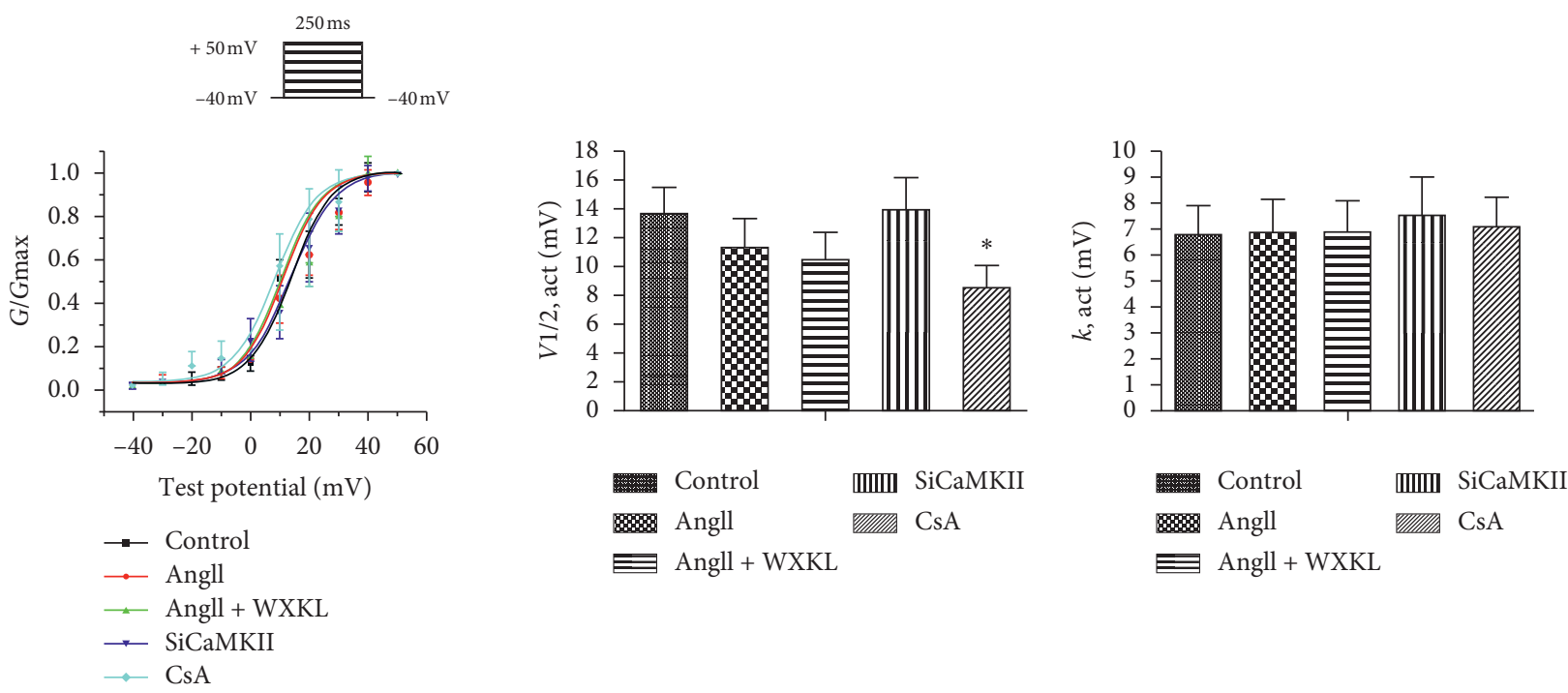

(c)

FIGURE 3: Continued. 


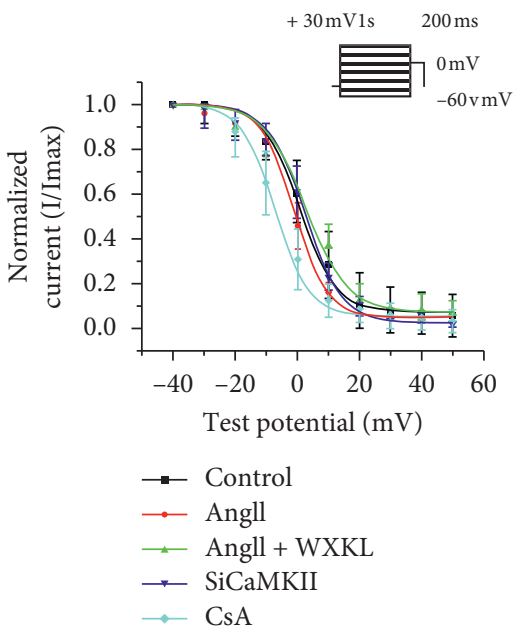

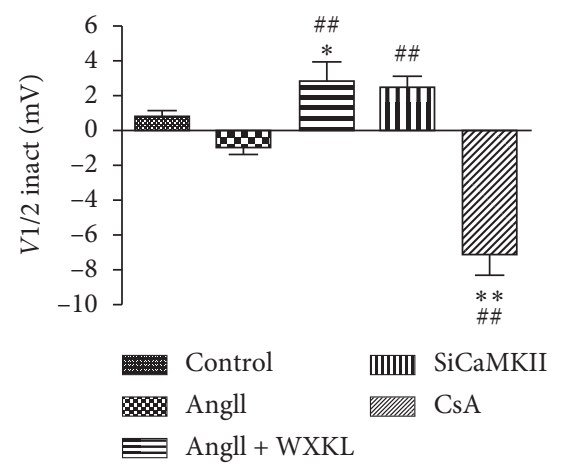

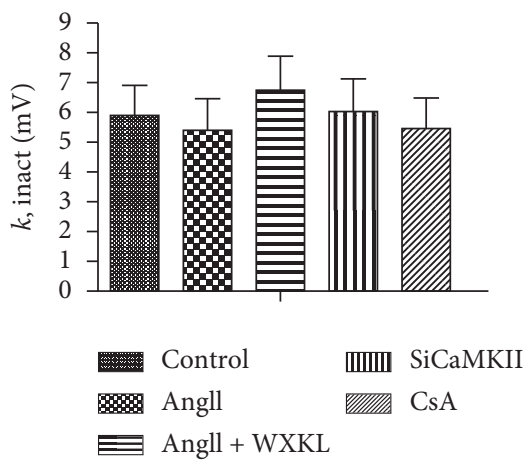

(d)

FIgUre 3: Changes in patch clamp inspection. (a) $\mathrm{I}_{\mathrm{Ca}-\mathrm{L}}$ current in the control, Ang II, Ang II + WXKL, SiCaMKII, and CsA groups. (b) Effects of siCaMKII and CsA on the $\mathrm{I}_{\mathrm{Ca}-\mathrm{L}}$ current-voltage (I-V) curve in H9c2 cells. I-V curves and peak current density (PA/PF) of each group ( $n=10$ cells per group). (c) Effects of siCaMKII and CsA on $\mathrm{I}_{\mathrm{Ca}-\mathrm{L}}$ steady-state activation (SSA) in H9c2 cells. $\mathrm{I}_{\mathrm{Ca}-\mathrm{L}}$ steady-state activation curves (original data point diagram and curve fit by Boltzmann equation), comparison of semiactivation voltage ( $V_{1 / 2}$, act), and comparison of slope factors ( $\left.\mathrm{K}_{\text {,act }}\right)$ in each group ( $n=10$ cells per group). (d) Effects of siCaMKII and CsA on $\mathrm{I}_{\mathrm{Ca}-\mathrm{L}}$ steady-state inactivation in H9c2 cells. $\mathrm{I}_{\mathrm{Ca}-\mathrm{L}}$ steady-state inactivation curves (original data point diagram and curve fitting by Boltzmann equation); comparison of semi-inactivation voltage $\left(V_{1 / 2}\right.$, inact $)$; comparison of slope factors $\left(\mathrm{K}_{\text {,inact }}\right)$ in each group $(n=10$ cells per group). Values are presented as mean \pm SD; Statistical significance was determined by one-way ANOVA. ${ }^{*} P<0.05$ and ${ }^{* *} P<0.01$ vs. the control group. ${ }^{\#} P<0.05$ and ${ }^{\# \#} P<0.01$ vs. the Ang II group.

protein expression decreased after that in the siCaMKII group. In the CsA group, NF- $\kappa \mathrm{B}$ expression was slightly decreased whereas that of other proteins was increased. After treatment with Ang II for $48 \mathrm{~h}$, the rate of increase of MyD88 and TLR2 was higher in the CsA group than that in H9c2 cells. After administering WXKL, protein expression decreased in each group. The previously mentioned findings indicated that, in the siCaMKII group, protein expression reduced in Ang II-induced cardiomyocytes and the inflammatory pathway was inhibited, but the opposite result was observed in the CsA group. Furthermore, the effect of WXKL was similar to that of siCaMKII.

\section{Discussion}

In the present study, we found that [1] Ang II activated the CaMKII, CnA-NFAT, and MyD88 inflammatory pathways in H9c2 cells and caused myocardial hypertrophy [2]. The siRNAmediated silencing of CaMKII inhibited protein expression in the CaMKII pathway, further attenuated protein expression in the CnA-NFAT signaling pathway, and inhibited the reduction in NFATc4 nuclear transfer. Moreover, CaMKII silencing played a role in improving Ang II-induced myocardial hypertrophy, and WXKL had a similar effect [3]. CsA, a CaN inhibitor, inhibited expression in the CnA-NFAT pathway but activated the CaMKII and MyD88 signaling pathways.

Both physiological and pathological $\mathrm{CH}$ are related to the increase in cardiomyocyte $\mathrm{Ca}^{2+}$ levels. $\mathrm{Ca}^{2+}$ regulates the activity of various $\mathrm{Ca}^{2+}$-dependent signaling pathways, including those of CaMKII and CnA-NFAT [6]
(Figure 9). It is known that CaMKII phosphorylates class II histone deacetylases (HDACs), particularly HDAC4 and HDAC5, by boosting the export of these molecules from the nucleus, resulting in the disinhibition of MEF-2mediated gene expression and $\mathrm{CH}$ [27-30]. $\mathrm{CnA}$ can maintain NFAT activity through a noncatalytic mechanism by associating with NFAT, blocking its nuclear export sequence and thereby maintaining its nuclear localization [31]. Once activated by sustained elevation of intracellular calcium, CaN dephosphorylates NFAT, enabling its translocation to the nucleus and consequently activating prohypertrophic target genes [32]. Our data showed that Ang II stimulation results in NFATc4 translocated to the nucleus, whereas pretreatment with siCaMKII or CsA inhibited Ang II-induced nuclear translocation of NFATc4; furthermore, transfer was inhibited after treatment with WXKL.

Previous research has shown that CaMKII inhibition can decrease Ang II-induced cardiac fibroblast proliferation and the secretion of TGF- $\beta 1$ and TNF- $\alpha$. In addition, CaMKII inhibition reversed the upregulation of MMP-1, 2, and 9 and collagen I and III after Ang II intervention [33]. A previous study revealed that treatment with KN-93 (a CaMKII inhibitor) significantly reduced the expression of $\mathrm{CH}$-related proteins, including NFATc3, p-HDAC4, p-HDAC5, GATA-4, and the hypertrophy marker BNP. Furthermore, the combined inhibition of the CaMKII and CaN signaling pathways may obviously relieve $\mathrm{CH}$ responses [13]. The results of the current study revealed that siCaMKII inhibited 

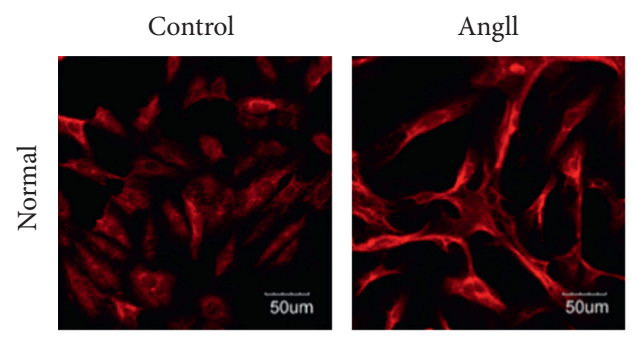

Angll + WXKL
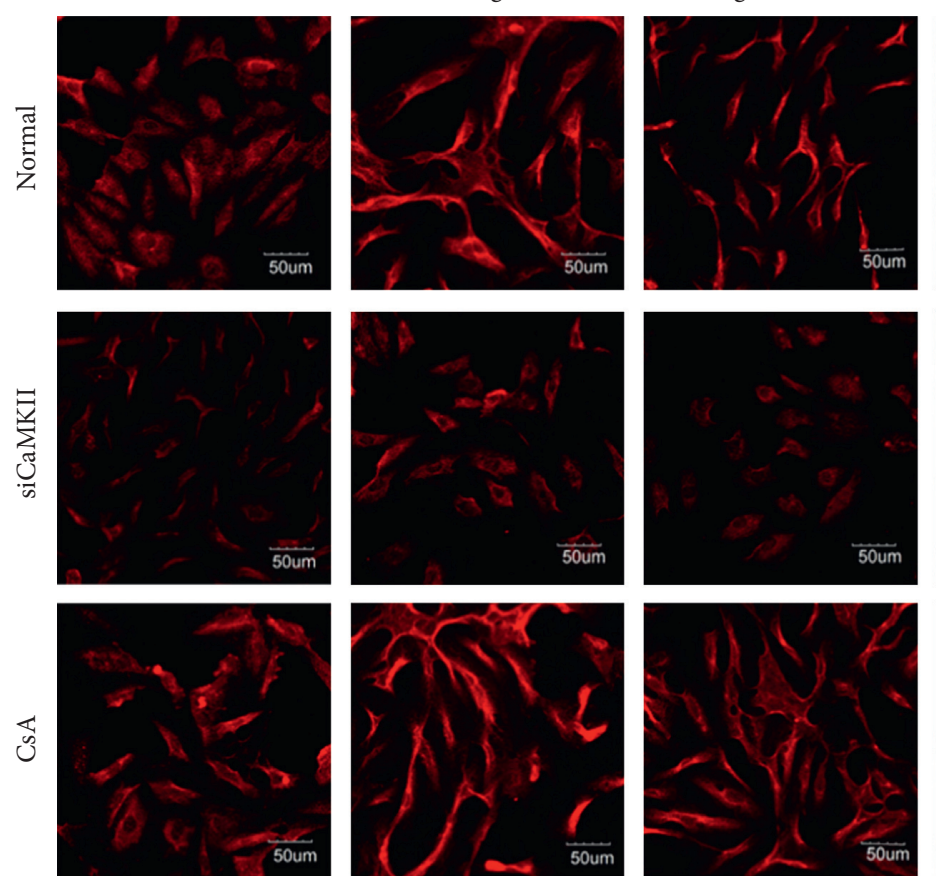
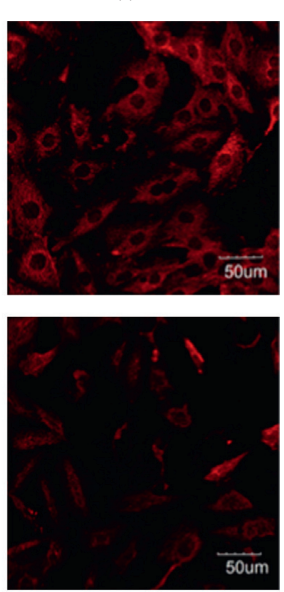

(a)

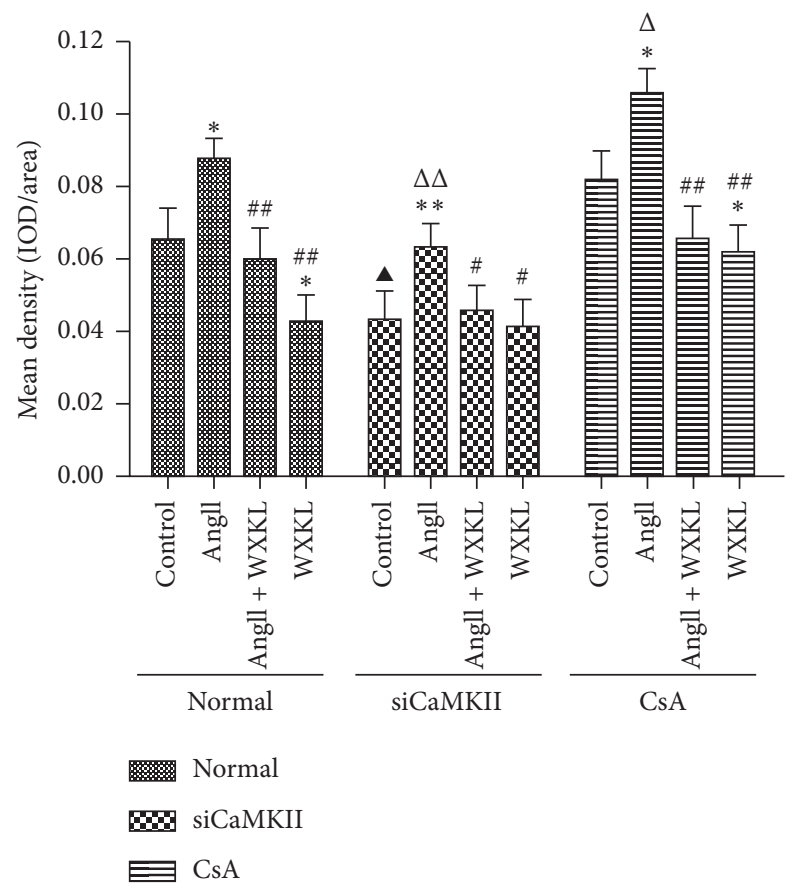

(b)

FIGURE 4: Effects of siCaMKII and CsA on the CaMKII signaling cascade. (a) Double immunofluorescence staining to observe the effects of siCaMKII, CsA, and WXKL on CaMKII expression in each group. Scale bar: $50 \mu \mathrm{m}$. (b) Mean density of CaMKII in each group. Data are presented as the mean $\pm \mathrm{SD}$. Statistical significance was determined by one-way ANOVA. ${ }^{*} P<0.05$ and ${ }^{* *} P<0.01$ vs. the control group. ${ }^{\#} P<0.05$ and ${ }^{\# \#} P<0.01$ vs. the Ang II group. ${ }^{\mathbf{\Delta}} P<0.05$ and ${ }^{\mathbf{\Lambda}} P<0.01$; the control group in SiCaMKII or CsA vs. the control group in normal. ${ }^{\triangle} P<0.05$ and ${ }^{\triangle}{ }^{\triangle} P<0.01$; the Ang II group in SiCaMKII or CsA vs. the Ang II group in normal.

protein expression in the CaMKII pathway and further reduced that in the CnA-NFAT signal pathway, thereby improving Ang II-induced hypertrophic cardiomyocytes. This was similar to the results of previous studies that demonstrated that, in the absence of CaMKII signals, CaN does not seem to contribute to abnormal cardiac remodeling, thus highlighting CaMKII and not $\mathrm{CaN}$ as a promising drug target to combat HF [16]. 


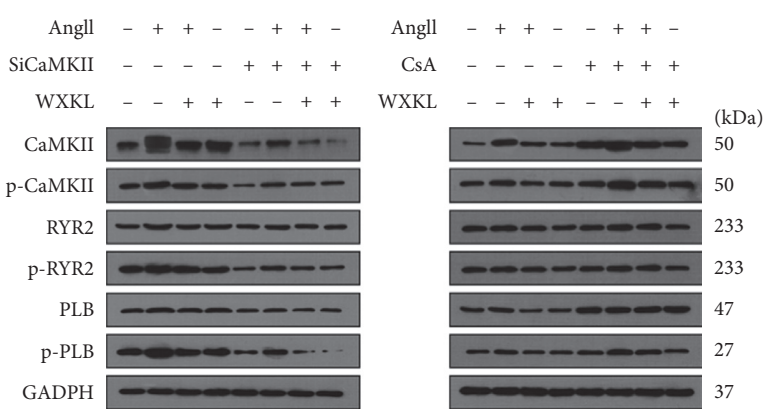

(a)

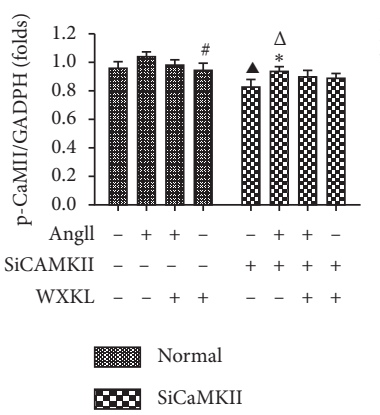

(c)

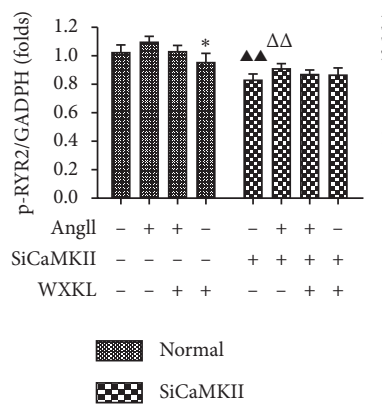

(e)

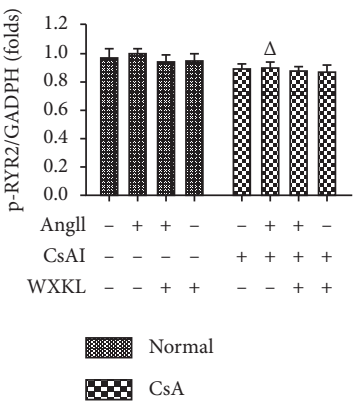

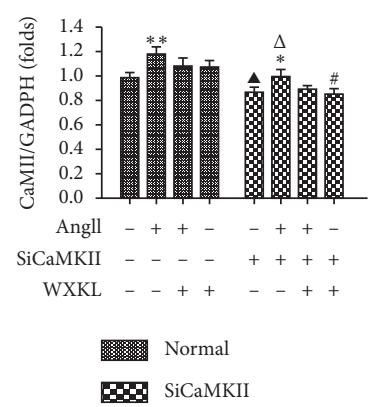

(b)

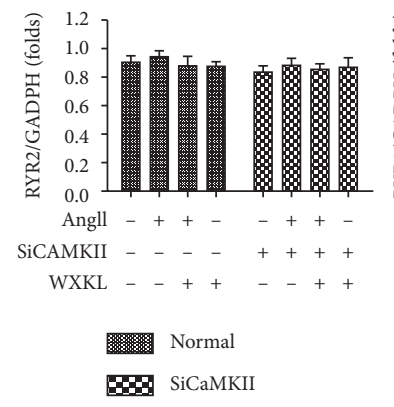

(d)

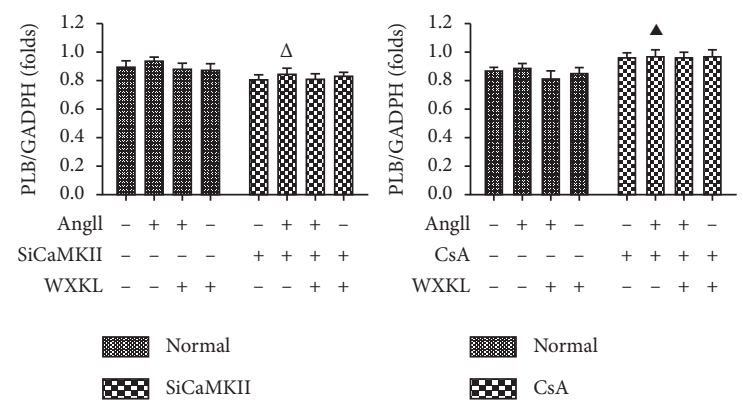

(f)
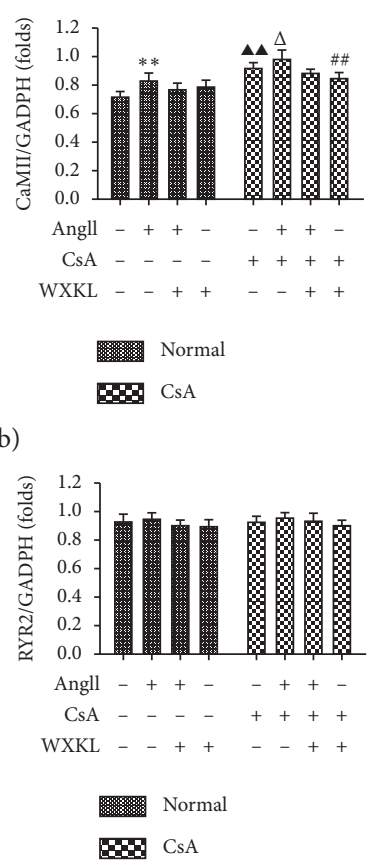

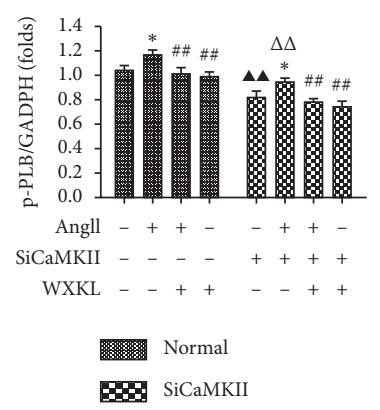

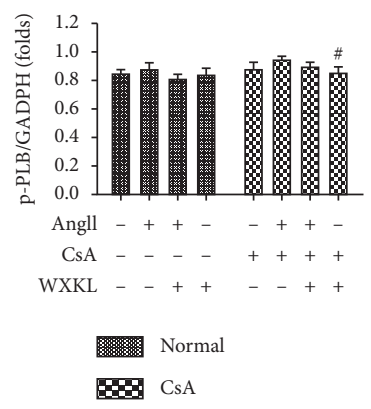

(g)

FIGURE 5: Effects of siCaMKII and CsA on Ang II-induced activation of CaMKII signaling in H9c2 cells. (a) Representative western blotting images. Densitometric analysis of (b) CaMKII, (c) p-CaMKII, (d) RyR2, (e) p-RyR2, (f) PLB, and (g) p-PLB expression levels ( $n=3$ cells per group). Data are presented as the mean \pm SD. Statistical significance was determined by one-way ANOVA. ${ }^{*} P<0.05$ and ${ }^{* *} P<0.01$ vs. the control group. ${ }^{\#} P<0.05$ and ${ }^{\# \#} P<0.01$ vs. the Ang II group. ${ }^{\boldsymbol{\Delta}} P<0.05$ and ${ }^{\boldsymbol{\Delta}} \boldsymbol{\Delta} P<0.01$; the control group in SiCaMKII or CsA vs. the control group in normal. ${ }^{\triangle} P<0.05$ and ${ }^{\triangle} \triangle P<0.01$; the Ang II group in SiCaMKII or CsA vs. the Ang II group in normal.

Excess CaMKII activity leads to phosphorylation of the L-type $\mathrm{Ca}^{2+}$ channel (LTCC), sarcoplasmic-endoplasmic reticulum $\mathrm{Ca}^{2+}$-ATPase, ryanodine receptor 2 (RyR2), and PLB proteins. CaMKII phosphorylates the LTCC that leads to increased $\mathrm{I}_{\mathrm{Ca}-\mathrm{L}}$ and forms a $\mathrm{Ca}^{2+}$ overload in the cell, which causes early afterdepolarizations and triggers arrhythmia. Hyperphosphorylation of the sarcoplasmic reticulum (SR) results in the consumption of $\mathrm{SR} \mathrm{Ca}^{2+}$ stores, leading to damaged cytosolic $\mathrm{Ca}^{2+}$ transients, which in turn induces systolic and diastolic dysfunction. Moreover, hyperphosphorylation events at RyR2 cause abnormal release of SR $\mathrm{Ca}^{2+}$ that activates the electrogenic $\mathrm{Na}^{+} / \mathrm{Ca}^{2+}$ - 

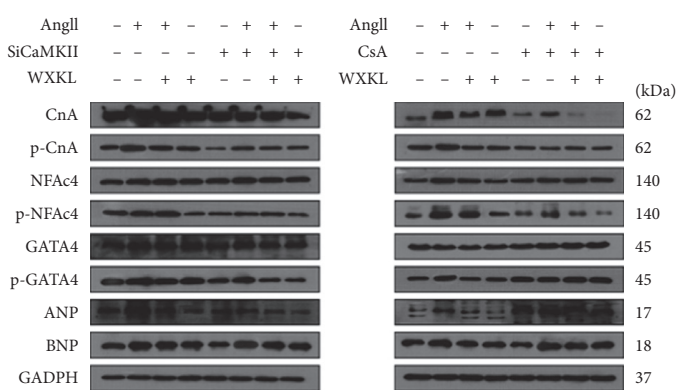

(a)

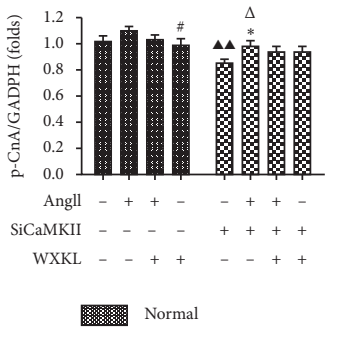

ES SiCaMKII

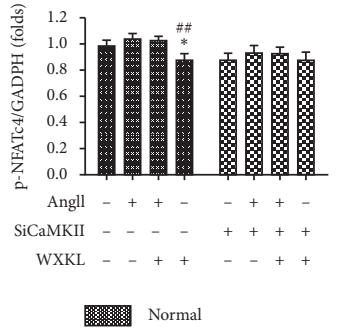

B8 SiCaMKII

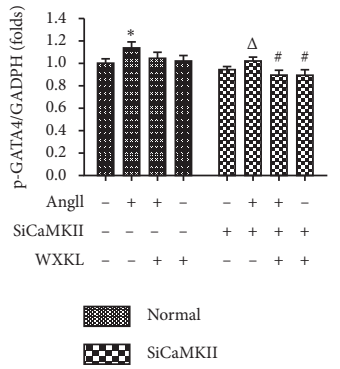

(e)

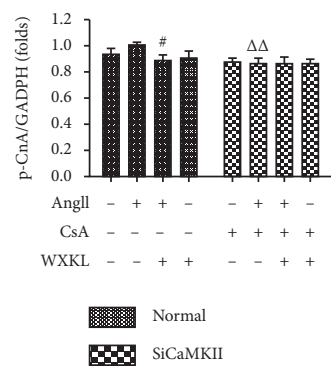

(c)
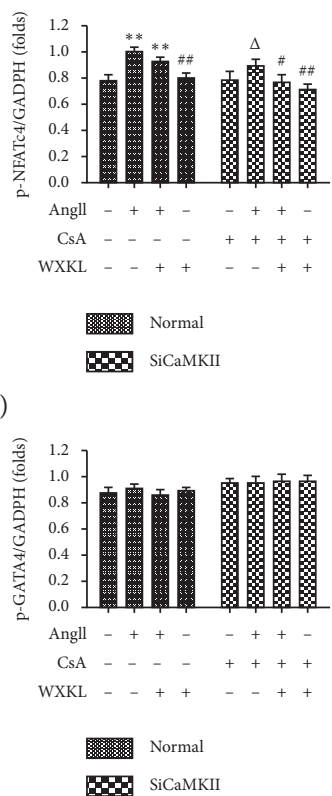

(g)

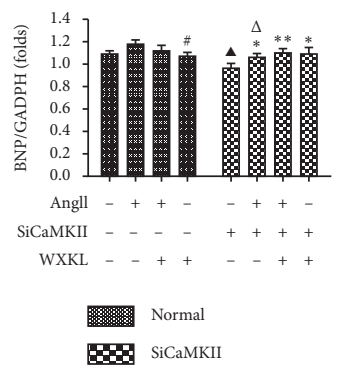

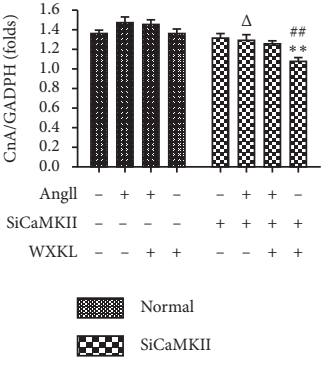

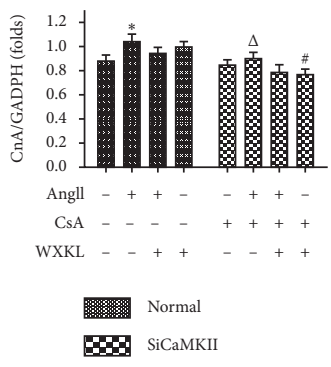

(b)
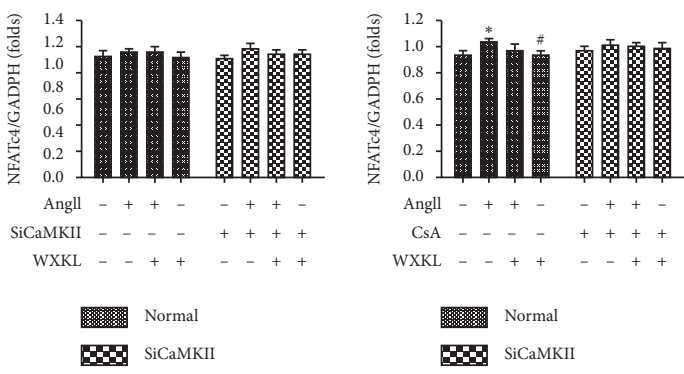

(d)
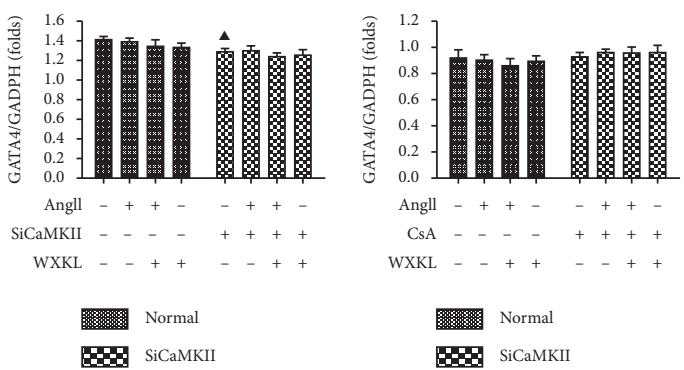

(f)
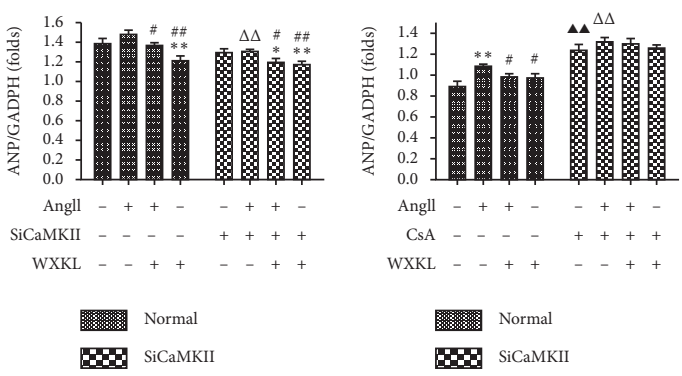

(h)

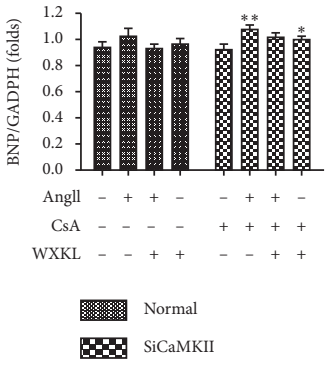

(i)

Figure 6: Effects of siCaMKII and CsA on Ang II-induced activation of CnA-NAFT signaling in H9c2 cells. (a) Representative western blotting images. Densitometric analysis of (b) CnA, (c) p-CnA, (d) NFATc4, (e) p-NFATc4, (f) GATA4, (g) p-GATA4, (h) ANP, and (i) BNP expression levels ( $n=3$ cells per group). Data are presented as the mean \pm SD. Statistical significance was determined by one-way ANOVA. ${ }^{*} P<0.05$ and ${ }^{* *} P<0.01$ vs. the control group. ${ }^{\#} P<0.05$ and ${ }^{\# \#} P<0.01$ vs. the Ang II group. ${ }^{\boldsymbol{\Delta}} P<0.05$ and $\boldsymbol{\Delta} \boldsymbol{\Delta} P<0.01$; the control group in SiCaMKII or CsA vs. the control group in normal. ${ }^{\triangle} P<0.05$ and ${ }^{\triangle} \triangle P<0.01$; the Ang II group in SiCaMKII or CsA vs. the Ang II group in normal. 


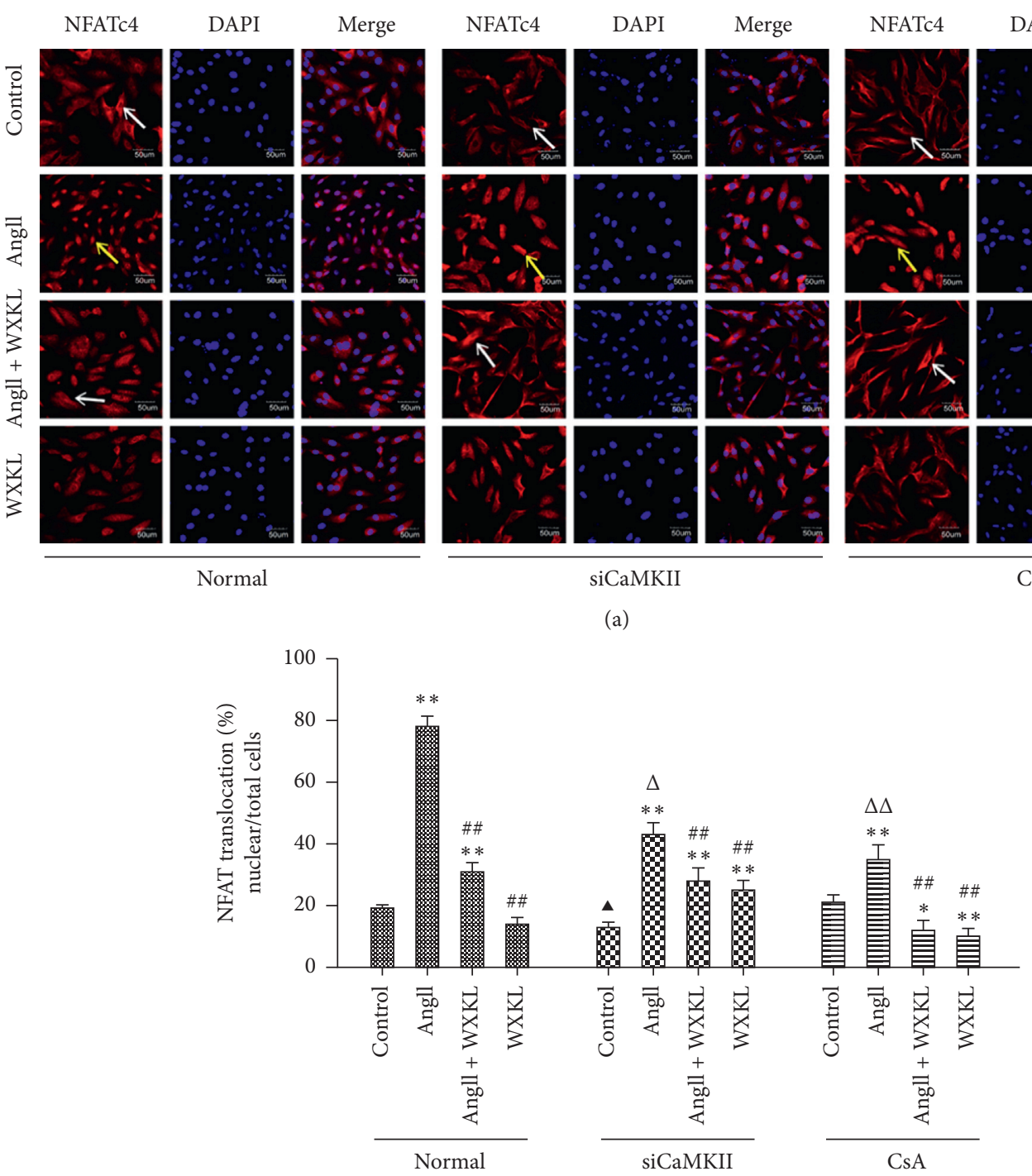

(b)

FIgURE 7: Ang II-induced NFATc4 nuclear translocation is inhibited by siCaMKII. H9c2 cells were treated with Ang II (10 $\left.{ }^{-7} \mathrm{M}\right)$ for $48 \mathrm{~h}$. CaMKII was silenced or CsA $\left(10^{-6} \mathrm{M}\right)$ was added to the culture medium prior to Ang II administration. WXKL (5 g/L) was added for $24 \mathrm{~h}$ after Ang II administration. The control cells received no treatment. (a) Representative images of immunofluorescence staining of NFATc4. Blue: DAPI staining; red: NFATc4. Scale bar: $50 \mu \mathrm{m}$. Yellow arrow: nuclear translocation; white arrow: no nuclear translocation or nuclear translocation was decreased. (b) Semiquantitative analysis of NFATc4 nuclear translocation. Data are presented as the mean \pm SD. Statistical significance was determined by one-way ANOVA. ${ }^{*} P<0.05$ and ${ }^{* *} P<0.01$ vs. the control group. ${ }^{\#} P<0.05$ and ${ }^{\# \#} P<0.01$ vs. the Ang II group. ${ }^{\wedge} P<0.05$ and ${ }^{\boldsymbol{\Delta}} \mathrm{\Delta} P<0.01$; the control group in SiCaMKII or CsA vs. the control group in normal. ${ }^{\triangle} P<0.05$ and ${ }^{\triangle \triangle} P<0.01 ;$ the Ang II group in SiCaMKII or CsA vs. the Ang II group in normal.

exchanger (NCX), which can cause delayed afterdepolarizations [34]. Interestingly, our study revealed that CnA expression was gradually reduced after treatment with CsA, but the CaMKII signaling pathway tended to be activated. This result was consistent with few studies that found either no effect or a deterioration of hypertrophy with CsA or FK506 [35, 36]. Conversely, previous studies have suggested that $\mathrm{CaN}$ inhibitors attenuate hypertrophy in a variety of models [37]. These discrepancies may be explained by the inherent differences between the different models. In addition, the inherent nonspecific effects associated with CsA and FK506 are to be considered. FK506 can also directly alter ryanodine receptor function through its effects on FKBP12 and FKBP12.6, while CsA may influence the leakage of calcium from the sarcoplasmic reticulum through the lipid bilayer $[38,39]$. Acute treatment with CsA causes alterations of LTCC activity in disassociated human cardiomyocytes [40]. The current study demonstrated that administration of CsA activated the CaMKII signaling pathway and increased the degree of $\mathrm{CH}$. This is possibly because the specificity of actions by CsA may be associated with their selective interactions with specific $\mathrm{CaN}$ subtypes or because of the presence of cell-specific CaN substrates [41]. 


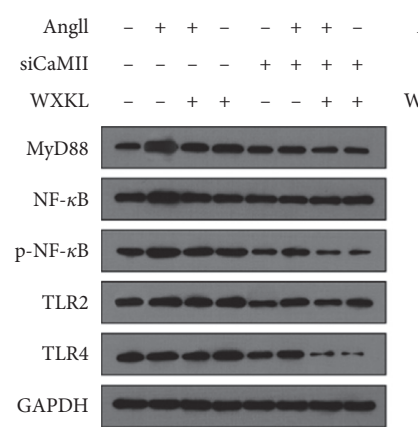

(a)

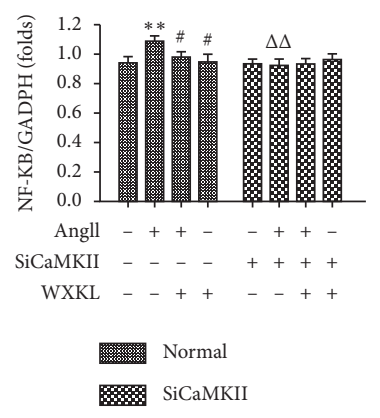

(c)

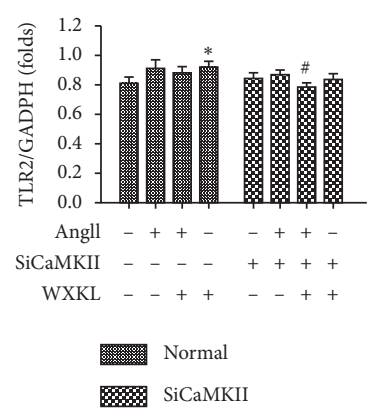

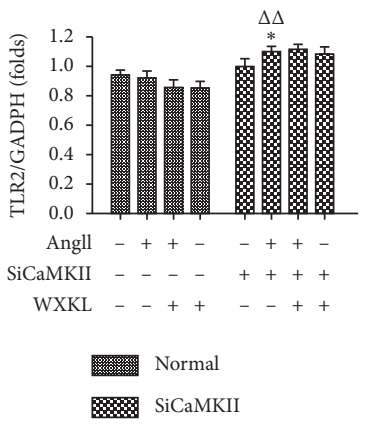

(e)
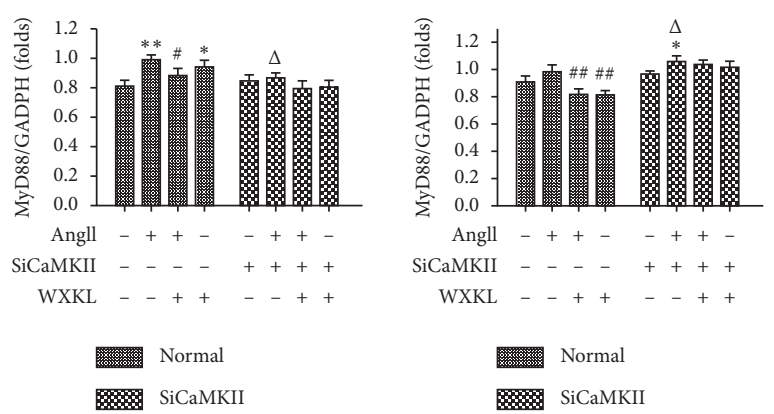

(b)
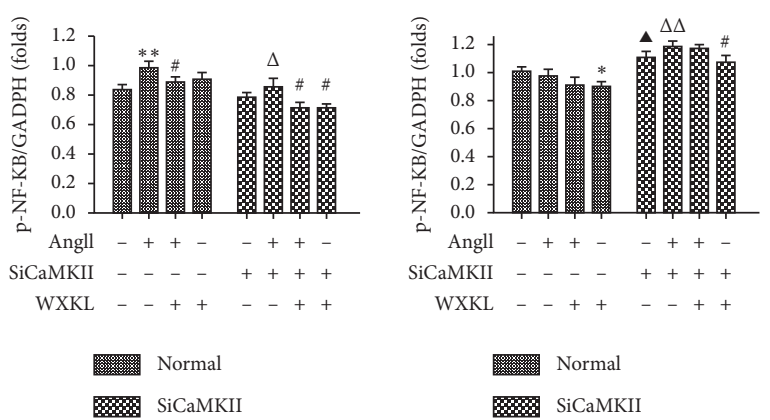

(d)
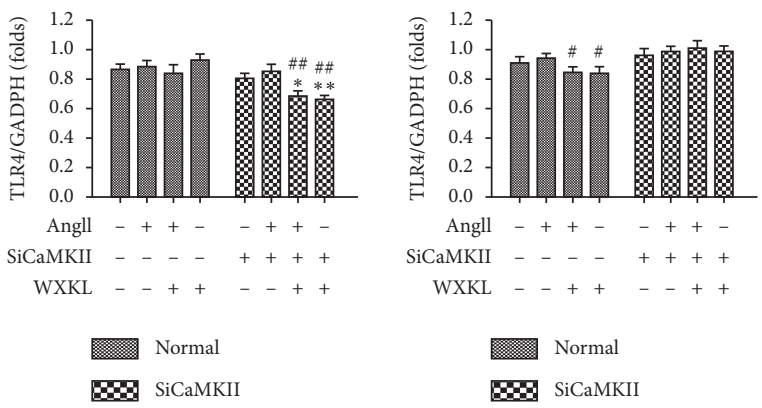

(f)

Figure 8: Effects of siCaMKII and CsA on Ang II-induced activation of the inflammatory signal transduction pathway in H9c2 cells. (a) Representative western blotting images. Densitometric analysis of (b) MyD88, (c) NF- $\kappa$ B, (d) p-NF- $\kappa B$, (e) TLR2, and (f) TLR4 expression levels $\left(n=3\right.$ cells per group). Data are presented as the mean \pm SD. Statistical significance was determined by one-way ANOVA. ${ }^{*} P<0.05$ and ${ }^{* *} P<0.01$ vs. the control group. ${ }^{\#} P<0.05$ and ${ }^{\#} P<0.01$ vs. the Ang II group. ${ }^{\mathbf{}} P<0.05$ and ${ }^{\mathbf{\Delta}} P<0.01$; the control group in SiCaMKII or CsA vs. the control group in normal. ${ }^{\triangle} P<0.05$ and ${ }^{\triangle} \triangle P<0.01$; the Ang II group in SiCaMKII or CsA vs. the Ang II group in normal.

Three different $\mathrm{CnA}$ subtypes are found in mammals: $\mathrm{CnA} \alpha$ and $\mathrm{CnA} \beta$, which are universally expressed, and $\mathrm{CnA} \gamma$ [9]. Furthermore, it has been reported that $\mathrm{CnA} \beta 1$ may have a cardioprotective action that decreases inflammation and scar formation [9]. Moreover, mice overexpressing constitutively active $\mathrm{CaN}$ show decreased apoptosis after ischemia/reperfusion, whereas deletion of the phosphatase-encoding exon of $\mathrm{CnA} \beta$ leads to increased cell death and reduced cardiac function $[42,43]$. In contrast, transgenic mice overexpressing an artificially truncated, constitutively active form of $\mathrm{CnA} \alpha$ lacking the autoinhibitory domain show strong $\mathrm{CH}$ and develop HF within the first few weeks of life, which is a response phenocopied by overexpression of a constitutively active form of NFAT [32]. Other studies have suggested that knockout mice lacking the phosphatase domain of $\operatorname{CnA} \beta$ show smaller hearts at baseline and exhibit reduced hypertrophy in response to pressure overload, Ang II, or isoproterenol [44]. Notably, in the study by Zhang et al. [36], CsA augmented hypertension but did not prevent $\mathrm{CH}$ in spontaneously hypertensive rats. CsA is known to cause numerous unwanted side effects. It has been shown that CsA increases Ang II receptors independently from CaN inhibition, which causes vasoconstriction and systemic hypertension and can promote $\mathrm{CH}[35,45-47]$. This may be due to CsA alleviating the cardioprotective action of $\mathrm{CnA} \beta$.

There have been some studies on the mechanism of CaMKII and inflammatory pathway regulation [48-53]. Increasing evidence has shown that as a multipurpose kinase, CaMKII plays a pivotal role in many cardiac pathophysiological conditions 


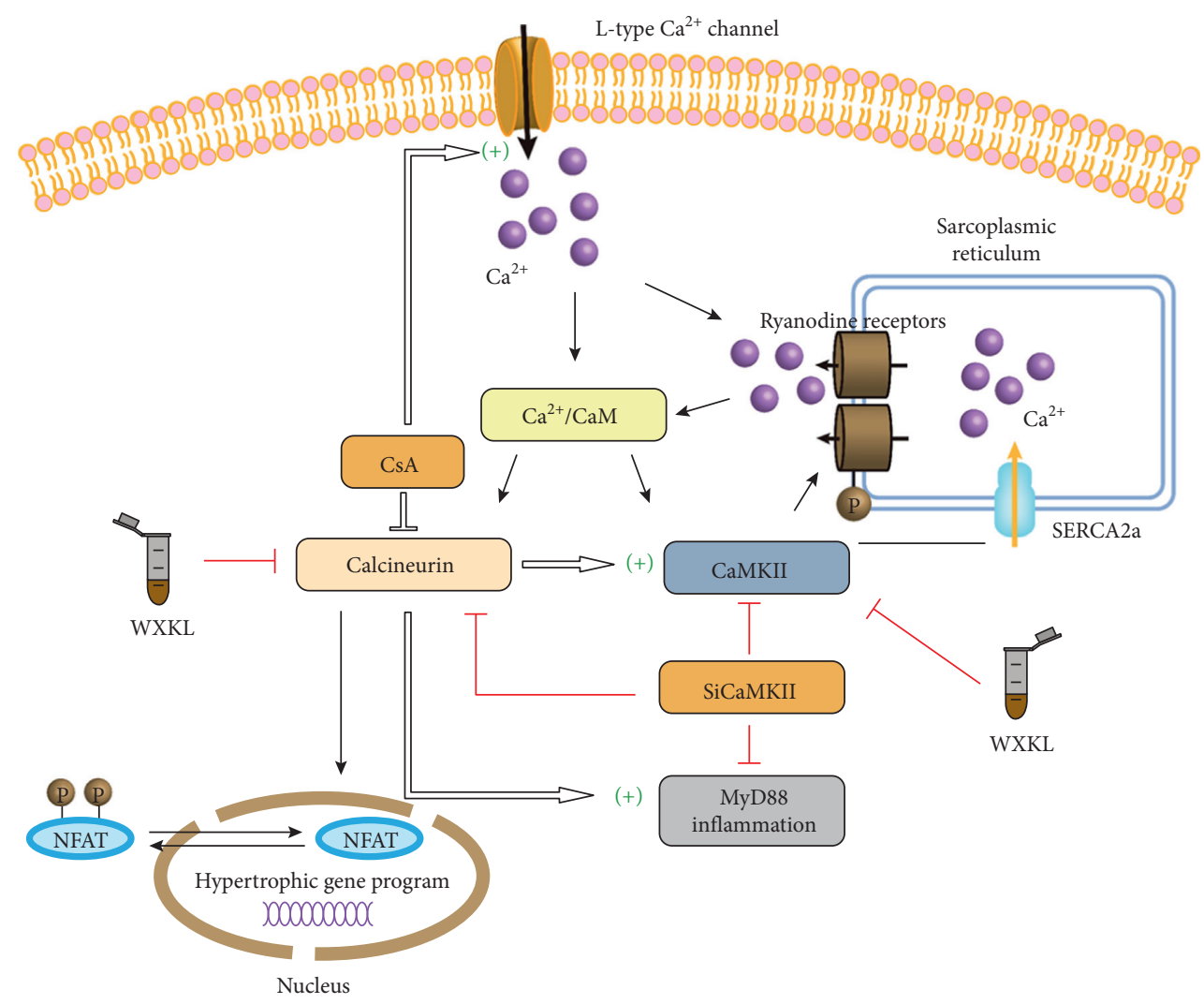

FIGURE 9: $\mathrm{Ca}^{2+}$-dependent signaling pathways in cardiac hypertrophy. Upon activation by CaM, CaN dephosphorylates cytoplasmic NFAT, a known hypertrophic transcription factor, and promotes its translocation to the nucleus and subsequent transcriptional activity. After silencing CaMKII, protein expression in the CaMKII and CaN-NFAT signaling pathways was inhibited, and the nuclear transfer of NFATc4 was decreased. CsA, an inhibitor of CaN, inhibits the expression in the CaN-NFAT pathway but activates the CaMKII signaling pathway, MyD88 inflammatory pathway, and $\mathrm{I}_{\mathrm{Ca}-\mathrm{L}}$. WXKL improves $\mathrm{CH}$ through the CaMKII and CaN-NFAT signaling pathways. CaM: calmodulin; CaN: calcineurin; NFAT: nuclear factor of activated T cells; CaMKII: $\mathrm{Ca}^{2+} /$ calmodulin-dependent protein kinase $\mathrm{II}$; $\mathrm{L}_{-}$type $\mathrm{Ca}^{2+}$ current: $\mathrm{I}_{\mathrm{Ca}}$ L; CsA: cyclosporine A; WXKL: Wenxin Keli; CH: cardiac hypertrophy.

involving inflammation [54]. Previous studies have shown that TLR4 mediates endothelial inflammation, activation, and dysfunction induced by $\mathrm{CaN}$ inhibitors, and according to previous observations, MyD88 silencing in endothelial cells prevented the induction of proinflammatory and endothelial activation markers by CsA and tacrolimus [55]. Our data indicate that, after CsA treatment, the expression of MyD88 and TLR2 obviously increased. The activation of MyD88, NF- $\kappa \mathrm{B}$, TLR2, and TLR4 inflammatory response pathways in pathological states induces an increase in CaMKII protein expression, causes calcium homeostasis, and promotes the occurrence of potentially malignant arrhythmias $[56,57]$.

WXKL is the first antiarrhythmic Chinese medicine to be approved by the state. Our previous studies indicated that WXKL treatment considerably preserves cardiac function and inhibits arrhythmia by modulating the CaMKII signaling pathway $[22,23]$. Moreover, WXKL treats $\mathrm{CH}$ and arrhythmia through a mechanism that possibly involves LTCC regulation [20]. We propose a new role for WXKL that may inhibit $\mathrm{CH}$ by regulating pathological autophagy [21]. Notably, treatment with WXKL significantly inhibited Ang II-induced hypertrophy in the present study. In addition, WXKL suppressed Ang II-induced elevated expression of CaMKII, CnA, and NFATc4 and prevented Ang II-induced nuclear translocation of NFATc4, suggesting that WXKL attenuated $\mathrm{CH}$ by inhibiting the CaMKII and CnA-NFAT signaling pathways. Therefore, owing to its numerous therapeutic benefits, WXKL may be considered as a promising choice for the treatment of $\mathrm{CH}$.

In conclusion, the present study demonstrated that siCaMKII attenuates Ang II-induced $\mathrm{CH}$, which may be partially associated with the downregulation of the CnANFAT and MyD88 signaling pathways, and WXKL had a similar effect. Furthermore, siCaMKII may be a promising approach to attenuate the progression of $\mathrm{CH}$ and arrhythmia. Our studies define the crosstalk between the CaMKII and CnA-NFAT signaling pathways in vitro, so numerous biological studies are needed for further research in vivo. This will improve the understanding of the mechanisms underlying hypertrophy and may provide evidence for drug application in the treatment of $\mathrm{CH}$.
Abbreviations
CaM:
Calcium/calmodulin
CaMKII: Calcium-/calmodulin-dependent protein kinase II 


$\begin{array}{ll}\text { CnA- } & \text { Calcineurin A-nuclear factor of activated } \\ \text { NFAT: } & \text { T cells } \\ \text { CH: } & \text { Cardiomyocyte hypertrophy } \\ \text { Ang: } & \text { Angiotensin } \\ \text { siCaMKII: } & \text { Silenced CaMKII } \delta \\ \text { CsA: } & \text { Cyclosporine A } \\ \text { WXKL: } & \text { Wenxin Keli } \\ \text { ICa-L: } & \text { L-type Ca }{ }^{2+} \text { current } \\ \text { HF: } & \text { Heart failure } \\ \text { CaN: } & \text { Calcineurin } \\ \text { HDAC: } & \text { Histone deacetylase } \\ \text { LTCC: } & \text { L-type Ca }{ }^{2+} \text { channel } \\ \text { RyR2: } & \text { Ryanodine receptor } 2 \\ \text { SR: } & \text { Sarcoplasmic reticulum } \\ \text { NCX: } & \text { Na }^{+} / \text {Ca }{ }^{2+} \text {-exchanger } \\ \text { BSA: } & \text { Bovine serum albumin } \\ \text { PBS: } & \text { Phosphate-buffered saline. }\end{array}$

\section{Data Availability}

The data and materials used in the study are available from the corresponding author upon reasonable request.

\section{Conflicts of Interest}

The authors declare that they have no conflicts of interest.

\section{Authors' Contributions}

XYW and GYH designed the work. AN wrote the article. CY contributed to cell experiment. XYF and WHH were responsible for critical revision of the article. GXY and CHW searched a lot of literature and revised the manuscript. SK and LYY contributed to the results interpretation. LXY, YF, and PXD analyzed the data. HXF, WX, and LY revised the manuscript.

\section{Acknowledgments}

This work was supported by the National Key R\&D Program of China (grant nos. 2018YFC1704900 and 2018YFC1704901), National Natural Science Foundation of China (grant nos. 81373835 and 81430098), Scientific Research Program of Outstanding Young Talents in Universities of Fujian Province ([2016] 23), and Scientific Research Startup Funding Project of Fujian Health College (no. 2016-1-2).

\section{Supplementary Materials}

Table S1: list of antibodies. Figure S1: effects of siCaMKII and CsA on the CnA-NAFT signaling cascade; double immunofluorescent staining to observe the effects of siCaMKII, $\mathrm{CsA}$ and WXKL on expression of $\mathrm{CnA}$ in each group. Figure S2: H9c2-1632: validation of CaMKII $\delta$ (Rat) 1632 site cell line after RNA interference. (Supplementary Materials)

\section{References}

[1] L. Schirone, M. Forte, S. Palmerio et al., "A review of the molecular mechanisms underlying the development and progression of cardiac remodeling," Oxidative Medicine and Cellular Longevity, vol. 2017, Article ID 3920195, 9 pages, 2017.

[2] H.-Y. Fang, M.-Y. Hung, Y.-M. Lin et al., "17 $\beta$-Estradiol and/ or estrogen receptor alpha signaling blocks protein phosphatase 1 mediated ISO induced cardiac hypertrophy," PLoS One, vol. 13, no. 5, Article ID e0196569, 2018.

[3] C. W. Yancy, M. Jessup, B. Bozkurt et al., "2013 ACCF/AHA guideline for the management of heart failure: executive summary: a report of the American College of cardiology foundation/American heart association task force on practice guidelines," Circulation, vol. 128, no. 16, pp. 1810-1852, 2013.

[4] P. Balakumar and G. Jagadeesh, "Multifarious molecular signaling cascades of cardiac hypertrophy: can the muddy waters be cleared?" Pharmacological Research, vol. 62, no. 5, pp. 365-383, 2010.

[5] M. A. Shibu, C.-H. Kuo, B.-C. Chen et al., "Oolong tea prevents cardiomyocyte loss against hypoxia by attenuating p-JNK mediated hypertrophy and enhancing P-IGF1R, p-akt, and p-Badser136 activity and by fortifying NRF2 antioxidation system," Environmental Toxicology, vol. 33, no. 2, pp. 220-233, 2018.

[6] I. Shimizu and T. Minamino, "Physiological and pathological cardiac hypertrophy," Journal of Molecular and Cellular Cardiology, vol. 97, pp. 245-262, 2016.

[7] J. J. Saucerman and D. M. Bers, "Calmodulin mediates differential sensitivity of CaMKII and calcineurin to local $\mathrm{Ca}^{2+}$ in cardiac myocytes," Biophysical Journal, vol. 95, no. 10, pp. 4597-4612, 2008.

[8] B. Sanna, O. F. Bueno, Y.-S. Dai, B. J. Wilkins, and J. D. Molkentin, "Direct and indirect interactions between calcineurin-NFAT and MEK1-extracellular signal-regulated kinase $1 / 2$ signaling pathways regulate cardiac gene expression and cellular growth," Molecular and Cellular Biology, vol. 25, no. 3, pp. 865-878, 2005.

[9] L. E. Felkin, T. Narita, R. Germack et al., "Calcineurin splicing variant calcineurin $A \beta 1$ improves cardiac function after myocardial infarction without inducing hypertrophy," Circulation, vol. 123, no. 24, pp. 2838-2847, 2011.

[10] J. Heineke and J. D. Molkentin, "Regulation of cardiac hypertrophy by intracellular signalling pathways," Nature Reviews Molecular Cell Biology, vol. 7, no. 8, pp. 589-600, 2006.

[11] B. Fiedler, S. M. Lohmann, A. Smolenski et al., "Inhibition of calcineurin-NFAT hypertrophy signaling by cGMP-dependent protein kinase type I in cardiac myocytes," Proceedings of the National Academy of Sciences, vol. 99, no. 17, pp. 11363-11368, 2002.

[12] Q. Liu, Y. Chen, M. Auger-Messier, and J. D. Molkentin, "Interaction between NF $\kappa \mathrm{B}$ and NFAT coordinates cardiac hypertrophy and pathological remodeling," Circulation Research, vol. 110, no. 8, pp. 1077-1086, 2012.

[13] C. H. Chen, J. W. Lin, C. Y. Huang et al., "The combined inhibition of the CaMKII $\delta$ and calcineurin signaling cascade attenuates IGF-IIR-induced cardiac hypertrophy," Journal of Cellular Physiology, vol. 235, no. 4, pp. 3539-3547, 2019.

[14] T. Zhang, E. N. Johnson, Y. Gu et al., "The cardiac-specific nuclear $\delta$ BIsoform of $\mathrm{Ca}^{2+} /$ calmodulin-dependent protein kinase II induces hypertrophy and dilated cardiomyopathy associated with increased protein phosphatase 2A activity," 
Journal of Biological Chemistry, vol. 277, no. 2, pp. 1261-1267, 2002.

[15] T. Zhang, L. S. Maier, N. D. Dalton et al., "The $\delta$ C isoform of CaMKII is activated in cardiac hypertrophy and induces dilated cardiomyopathy and heart failure," Circulation Research, vol. 92, no. 8, pp. 912-919, 2003.

[16] M. M. Kreusser, L. H. Lehmann, S. Keranov et al., "Cardiac CaM kinase II genes $\delta$ and $\gamma$ contribute to adverse remodeling but redundantly inhibit calcineurin-induced myocardial hypertrophy," Circulation, vol. 130, no. 15, pp. 1262-1273, 2014.

[17] China Medical Science, Pharmacopoeia: The Pharmacopoeia Of the People's Republic of China, China Medical Science Press, Beijing, China, 2015.

[18] G. Nan, C. L. Zhang, C. Wang, C. Wang, Y. F. Zhu, and H. H. Wu, "Determination of notoginsenoside R1, ginsenoside Rg1, Rb1, Rd and lobetyolin in Wenxin Keli by HPLC," Tianjin Journal of Traditional Chinese Medicine, vol. 33, no. 7, pp. 434-436, 2016, in Chinese.

[19] Y. Chen, S. Nie, H. Gao et al., "The effects of Wenxin Keli on $P$-wave dispersion and maintenance of sinus rhythm in patients with paroxysmal atrial fibrillation: a meta-analysis of randomized controlled trials," Evidence-Based Complementary and Alternative Medicine, vol. 2013, Article ID 245958, 9 pages, 2013

[20] Y. Chen, Y. Li, L. Guo et al., "Effects of wenxin keli on the action potential and L-type calcium current in rats with transverse aortic constriction-induced heart failure," Evidence-Based Complementary and Alternative Medicine, vol. 2013, Article ID 572078, 12 pages, 2013.

[21] J. Li, Y. Li, Y. Zhang et al., "The inhibitory effect of WenxinKeli on H9c2 cardiomyocytes hypertrophy induced by angiotensin II through regulating autophagy activity," Oxidative Medicine and Cellular Longevity, vol. 2017, Article ID 7042872, 11 pages, 2017.

[22] X. Yang, Y. Chen, Y. Li, X. Ren, Y. Xing, and H. Shang, "Effects of Wenxin Keli on cardiac hypertrophy and arrhythmia via regulation of the calcium/calmodulin dependent kinase II signaling pathway," BioMed Research International, vol. 2017, Article ID 1569235, 12 pages, 2017.

[23] Y. Xing, Y. Gao, J. Chen et al., "Wenxin-Keli regulates the calcium/calmodulin-dependent protein kinase II signal transduction pathway and inhibits cardiac arrhythmia in rats with myocardial infarction," Evidence-Based Complementary and Alternative Medicine, vol. 2013, Article ID 464508, 15 pages, 2013.

[24] A.-M. Sun, "Effect of Wenxin granules combined with metoprolol on cardiac function and exercise tolerance in patients with coronary heart disease tachyarrhythmia," Chinese Journal of School Doctor, vol. 34, no. 7, pp. 502-504, 2020.

[25] F. Wang and Z.-Q. Chen, "Treatment effect of wenxin granules on patients with heart failure and atrial fibrillation," Practical Clinical Journal of Integrated Traditional Chinese and Western Medicine, vol. 19, no. 10, pp. 132-133, 2019.

[26] H.-E. Liu, "Curative effect of wenxin granule combined with metoprolol on patients with CHD and arrhythmia and and its effects on $24 \mathrm{~h}$ dynamic electrocardiogram," Journal of Rare and Uncommon Diseases, vol. 27, no. 4, pp. 37-39, 2020.

[27] J. Bossuyt, K. Helmstadter, X. Wu et al., " $\mathrm{Ca}^{2+} /$ Calmodulindependent protein kinase II $\delta$ and protein kinase D overexpression reinforce the histone deacetylase 5 redistribution in heart failure," Circulation Research, vol. 102, no. 6, pp. 695-702, 2008.

[28] J. Backs, K. Song, S. Bezprozvannaya, S. Chang, and E. N. Olson, "CaM kinase II selectively signals to histone deacetylase 4 during cardiomyocyte hypertrophy," Journal of Clinical Investigation, vol. 116, no. 7, pp. 1853-1864, 2006.

[29] R. Passier, H. Zeng, N. Frey et al., "CaM kinase signaling induces cardiac hypertrophy and activates the MEF2 transcription factor in vivo," Journal of Clinical Investigation, vol. 105, no. 10, pp. 1395-1406, 2000.

[30] C. L. Zhang, T. A. McKinsey, S. Chang, C. L. Antos, J. A. Hill, and E. N. Olson, "Class II histone deacetylases act as signalresponsive repressors of cardiac hypertrophy," Cell, vol. 110, no. 4, pp. 479-488, 2002.

[31] D. R. Higazi, C. J. Fearnley, F. M. Drawnel et al., "Endothelin1-stimulated InsP3-induced $\mathrm{Ca}^{2+}$ release is a nexus for hypertrophic signaling in cardiac myocytes," Molecular Cell, vol. 33, no. 4, pp. 472-482, 2009.

[32] J. D. Molkentin, J.-R. Lu, C. L. Antos et al., "A calcineurindependent transcriptional pathway for cardiac hypertrophy," Cell, vol. 93, no. 2, pp. 215-228, 1998.

[33] W. Zhang, D.-Q. Chen, F. Qi, J. Wang, W.-Y. Xiao, and W.-Z. Zhu, "Inhibition of calcium-calmodulin-dependent kinase II suppresses cardiac fibroblast proliferation and extracellular matrix secretion," Journal of Cardiovascular Pharmacology, vol. 55, no. 1, pp. 96-105, 2010.

[34] J. Mustroph, S. Neef, and L. S. Maier, "CaMKII as a target for arrhythmia suppression," Pharmacology \& Therapeutics, vol. 176, pp. 22-31, 2017.

[35] W. Hayashida, Y. Kihara, A. Yasaka, and S. Sasayama, "Cardiac calcineurin during transition from hypertrophy to heart failure in rats," Biochemical and Biophysical Research Communications, vol. 273, no. 1, pp. 347-351, 2000.

[36] W. Zhang, R. C. Kowal, F. Rusnak, R. A. Sikkink, E. N. Olson, and R. G. Victor, "Failure of calcineurin inhibitors to prevent pressure-overload left ventricular hypertrophy in rats," Circulation Research, vol. 84, no. 6, pp. 722-728, 1999.

[37] B. J. Wilkins and J. D. Molkentin, "Calcium-calcineurin signaling in the regulation of cardiac hypertrophy," Biochemical and Biophysical Research Communications, vol. 322, no. 4, pp. 1178-1191, 2004.

[38] K. S. Park, T. K. Kim, and D. H. Kim, "Cyclosporin A treatment alters characteristics of $\mathrm{Ca}^{2+}$-release channel in cardiac sarcoplasmic reticulum," American Journal of Physiology-Heart and Circulatory Physiology, vol. 276, no. 3, pp. H865-H872, 1999.

[39] A. Bandyopadhyay, D. W. Shin, J. O. Ahn, and D. H. Kim, "Calcineurin regulates ryanodine receptor $/ \mathrm{Ca}\left({ }^{2+}\right)$-release channels in rat heart," Biochemical Journal, vol. 352, no. 1, pp. 61-70, 2000.

[40] J. Matthes, A. Jager, R. Handrock et al., " $\mathrm{Ca}^{2+}$-dependent modulation of single human cardiac L-type calcium channels by the calcineurin inhibitor cyclosporine," Journal of Molecular and Cellular Cardiology, vol. 36, no. 2, pp. 241-255, 2004.

[41] J. Liu, J. D. Farmer Jr., W. S. Lane, J. Friedman, I. Weissman, and S. L. Schreiber, "Calcineurin is a common target of cyclophilin-cyclosporin A and FKBP-FK506 complexes," Cell, vol. 66, no. 4, pp. 807-815, 1991.

[42] L. J. De Windt, H. W. Lim, T. Taigen et al., "Calcineurinmediated hypertrophy protects cardiomyocytes from apoptosis in vitro and in vivo: an apoptosis-independent model of dilated heart failure," Circulation Research, vol. 86, no. 3, pp. 255-263, 2000.

[43] O. F. Bueno, D. J. Lips, R. A. Kaiser et al., "Calcineurin A $\beta$ gene targeting predisposes the myocardium to acute ischemia-induced apoptosis and dysfunction," Circulation Research, vol. 94, no. 1, pp. 91-99, 2004. 
[44] O. F. Bueno, B. J. Wilkins, K. M. Tymitz et al., "Impaired cardiac hypertrophic response in calcineurin A -deficient mice," Proceedings of the National Academy of Sciences, vol. 99, no. 7, pp. 4586-4591, 2002.

[45] A. D. Kirk, L. M. Jacobson, D. M. Heisey, N. A. Fass, H. W. Sollinger, and J. D. Pirsch, "Posttransplant diastolic hypertension: associations with intragraft transforming growth factor-beta, endothelin, and renin transcription," Transplantation, vol. 64, no. 12, pp. 1716-1720, 1997.

[46] P. V. Avdonin, F. Cottet-Maire, G. V. Afanasjeva, S. A. Loktionova, P. Lhote, and U. T. Ruegg, "Cyclosporine A up-regulates angiotensin II receptors and calcium responses in human vascular smooth muscle cells," Kidney International, vol. 55, no. 6, pp. 2407-2414, 1999.

[47] J. Sadoshima and S. Izumo, "Molecular characterization of angiotensin II--induced hypertrophy of cardiac myocytes and hyperplasia of cardiac fibroblasts: critical role of the AT1 receptor subtype," Circulation Research, vol. 73, no. 3, pp. 413-423, 1993.

[48] K. Kashiwase, Y. Higuchi, S. Hirotani et al., "CaMKII activates ASK1 and NF- $\kappa \mathrm{B}$ to induce cardiomyocyte hypertrophy," Biochemical and Biophysical Research Communications, vol. 327, no. 1, pp. 136-142, 2005.

[49] G. Liu, J. Zhao, Z. Chang, and G. Guo, "CaMKII activates ASK1 to induce apoptosis of spinal astrocytes under oxygenglucose deprivation," Cellular and Molecular Neurobiology, vol. 33, no. 4, pp. 543-549, 2013.

[50] J. Backs, T. Backs, S. Neef et al., "The isoform of CaM kinase II is required for pathological cardiac hypertrophy and remodeling after pressure overload," Proceedings of the $\mathrm{Na}$ tional Academy of Sciences, vol. 106, no. 7, pp. 2342-2347, 2009.

[51] H. Ling, C. B. B. Gray, A. C. Zambon et al., " $\mathrm{Ca}^{2+} / \mathrm{Cal}-$ modulin-dependent protein kinase II $\delta$ mediates myocardial ischemia/reperfusion injury through nuclear factor- $\kappa \mathrm{b}$," Circulation Research, vol. 112, no. 6, pp. 935-944, 2013.

[52] M. V. Singh, A. Kapoun, L. Higgins et al., " $\mathrm{Ca}^{2+} /$ calmodulindependent kinase II triggers cell membrane injury by inducing complement factor B gene expression in the mouse heart," Journal of Clinical Investigation, vol. 119, no. 4, pp. 986-996, 2009.

[53] X. Liu, M. Yao, N. Li, C. Wang, Y. Zheng, and X. Cao, "CaMKII promotes TLR-triggered proinflammatory cytokine and type I interferon production by directly binding and activating TAK1 and IRF3 in macrophages," Blood, vol. 112, no. 13, pp. 4961-4970, 2008.

[54] M. R. Rusciano, E. Sommariva, V. Douin-Echinard, M. Ciccarelli, P. Poggio, and A. S. Maione, "CaMKII activity in the inflammatory response of cardiac diseases," International Journal of Molecular Sciences, vol. 20, no. 18, p. 4374, 2019.

[55] R. Rodrigues-Diez, C. González-Guerrero, C. Ocaña-Salceda et al., "Calcineurin inhibitors cyclosporine A and tacrolimus induce vascular inflammation and endothelial activation through TLR4 signaling," Scientific Reports, vol. 6, no. 1, p. 27915, 2016.

[56] M. V. Singh, P. D. Swaminathan, E. D. Luczak, W. Kutschke, R. M. Weiss, and M. E. Anderson, "MyD88 mediated inflammatory signaling leads to CaMKII oxidation, cardiac hypertrophy and death after myocardial infarction," Journal of Molecular and Cellular Cardiology, vol. 52, no. 5, pp. 1135-1144, 2012.
[57] M. V. Singh and M. E. Anderson, "Is CaMKII a link between inflammation and hypertrophy in heart?" Journal of Molecular Medicine, vol. 89, no. 6, pp. 537-543, 2011. 